\title{
Linear MMSE-Optimal Turbo Equalization Using Context Trees
}

\author{
Kyeongyeon Kim, Member, IEEE, Nargiz Kalantarova, Suleyman Serdar Kozat, Senior Member, IEEE, and \\ Andrew C. Singer, Fellow, IEEE
}

\begin{abstract}
Formulations of the turbo equalization approach to iterative equalization and decoding vary greatly when channel knowledge is either partially or completely unknown. Maximum aposteriori probability (MAP) and minimum mean-square error (MMSE) approaches leverage channel knowledge to make explicit use of soft information (priors over the transmitted data bits) in a manner that is distinctly nonlinear, appearing either in a trellis formulation (MAP) or inside an inverted matrix (MMSE). To date, nearly all adaptive turbo equalization methods either estimate the channel or use a direct adaptation equalizer in which estimates of the transmitted data are formed from an expressly linear function of the received data and soft information, with this latter formulation being most common. We study a class of direct adaptation turbo equalizers that are both adaptive and nonlinear functions of the soft information from the decoder. We introduce piecewise linear models based on context trees that can adaptively approximate the nonlinear dependence of the equalizer on the soft information such that it can choose both the partition regions as well as the locally linear equalizer coefficients in each region independently, with computational complexity that remains of the order of a traditional direct adaptive linear equalizer. This approach is guaranteed to asymptotically achieve the performance of the best piecewise linear equalizer, and we quantify the MSE performance of the resulting algorithm and the convergence of its MSE to that of the linear minimum MSE estimator as the depth of the context tree and the data length increase.
\end{abstract}

Index Terms-Context tree, decision feedback, nonlinear equalization, piecewise linear, turbo equalization.

\section{INTRODUCTION}

I TERATIVE equalization and decoding methods, or so-called turbo equalization [1]-[3], have become increasingly popular methods for leveraging the power of forward error correction to enhance the performance of digital communication systems in which intersymbol interference or multiple access interference are present. Given full channel knowledge,

Manuscript received February 11, 2012; revised September 14, 2012 and February 19, 2013; accepted February 25, 2013. Date of publication April 04, 2013; date of current version May 20,2013. The associate editor coordinating the review of this manuscript and approving it for publication was Prof. Xiao-Ping Zhang.

K. Kim is with Samsung Electronics, Yongin-si, Gyeonggi-do 446-712, Korea (e-mail: adrianakky@gmail.com).

N. Kalantarova is with the Electrical Engineering Department, Koc University, Istanbul 34450, Turkey (e-mail: nkalantarova@ku.edu.tr).

S. S. Kozat is with the Electrical Engineering Department, Bilkent University, Ankara 06800, Turkey (e-mail: kozat@ee.bilkent.edu.tr).

A. C. Singer is with the Electrical and Computer Engineering Department at University of Illinois at Urbana-Champaign, Urbana, IL 61801, USA (e-mail: acsinger@illinois.edu).

Color versions of one or more of the figures in this paper are available online at http://ieeexplore.ieee.org.

Digital Object Identifier 10.1109/TSP.2013.2256899 maximum a posteriori probability (MAP) equalization and decoding give rise to an elegant manner in which the equalization and decoding problems can be (approximately) jointly resolved [1]. For large signal constellations or when the channel has a large delay spread resulting in substantial intersymbol interference, this approach becomes computationally prohibitive and lower complexity linear equalization strategies are often employed [4]-[6]. Computational complexity issues are also exacerbated by the use of multi-input/multi-output (MIMO) transmission strategies. It is important to note that MAP and MMSE formulations of the equalization component in such iterative receivers make explicit use of soft information from the decoder that is a nonlinear function of both the channel response and the soft information [5], which can be efficiently calculated for certain configurations [7]. In a MAP receiver, soft information is used to weight branch metrics in the receiver trellis [8]. In an MMSE receiver, this soft information is used in the (recursive) computation of the filter coefficients and appears inside of a matrix that is inverted [5].

In practice, most communication systems lack precise channel knowledge and must make use of pilots or other means to estimate and track the channel if the MAP or MMSE formulations of turbo equalization are to be used [5], [8]. Increasingly, however, receivers based on direct adaptation methods are used for the equalization component, due to their attractive computational complexity [4], [9], [10]. Specifically, the channel response is neither needed nor estimated for direct adaptation equalizers, since the transmitted data symbols are directly estimated based on the signals received. This is often accomplished with a linear or decision feedback structure that has linear complexity in the channel memory, as opposed to the quadratic complexity of the MMSE formulation, and is invariant to the constellation size [9]. A MAP receiver not only needs a channel estimate, but also has complexity that is exponential in the channel memory, where the base of the exponent is the transmit constellation size [8]. For example, underwater acoustic communications links often have a delay spread in excess of several tens to hundreds of symbol periods, make use of 4 or 16 QAM signal constellations, and have multiple transmitters and receive hydrophones [10], [11]. In our experience, for such underwater acoustic channels, MAP-based turbo equalization is infeasible and MMSE-based methods are impractical for all but the most benign channel conditions [10]. As such, direct-adaptation receivers that form an estimate of the transmitted symbols as a linear function of the received data, past decided symbols, and soft information from the decoder have emerged as the most pragmatic solution. Least-mean square (LMS)-based receivers are used in practice to estimate and track the filter coefficients in these soft-input/soft-output decision feedback equalizer structures, 
which are often multi-channel receivers for both SIMO and MIMO transmissions [4], [6], [9].

While such linear complexity receivers have addressed the computational complexity issues that make MAP and MMSE formulations unattractive or infeasible, they have also unduly restricted the potential benefit of incorporating soft information into the equalizer. Although such adaptive linear methods may converge to their "optimal", i.e., Wiener solution, they usually deliver inferior performance compared to a linear MMSE turbo receiver [12], since the Wiener solution for this stationarized problem, replaces the time-varying soft information by its time average [5], [13]. It is inherent in the structure of such adaptive approaches that an implicit assumption is made that the random process governing the received data and that of the soft-information sequence are both mean ergodic so that ensemble averages associated with the signal and soft information can be estimated with time averages. The primary source of performance loss of these adaptive algorithms is due to their implicit use of the log likelihood ratio (LLR) information from the decoder as stationary soft decision sequence [12], whereas a linear MMSE turbo equalizer considers this LLR information as nonstationary a priori statistics over the transmitted symbols [5].

Indeed, one of the strengths of the linear MMSE turbo equalizer lies in its ability to employ a distinctly different linear equalizer for each transmitted symbol [5], [6]. This arises from the time-varying nature of the local soft information available to the receiver from the decoder. Hence, even if the channel response were known and fixed (i.e., time-invariant), the MMSE-optimal linear turbo equalizer corresponds to a set of linear filter coefficients that are different for each and every transmitted symbol [5], [14]. This is due to the presence of the soft information inside an inverted matrix that is used to construct the MMSE-optimal equalizer coefficients. As a result, a time-invariant channel will still give rise to a recursive formulation of the equalizer coefficients that require quadratic complexity per output symbol. As an example in Fig. 1, we plot for a time invariant channel the time varying filter coefficients of the MMSE linear turbo equalizer, along with the filter coefficients of an LMS-based, direct adaptation turbo equalizer that has converged to its time invariant solution. This behavior is actually manifested due to the nonlinear relationship between the soft information and the MMSE filter coefficients.

In this paper, we explore a class of equalizers that maintain the linear complexity adaptation of linear, direct adaptation equalizers [9], but attempt to circumvent the loss of this nonlinear dependence of the MMSE optimal equalizer on the soft information from the decoder [5]. Specifically, we investigate an adaptive, piecewise linear model based on context trees [15] that partition the space of soft information from the decoder, such that locally linear (in soft information space) models may be used. However instead of using a fixed piecewise linear equalizer, the nonlinear algorithm we introduce can adaptively choose the partitioning of the space of soft information as well as the locally linear equalizer coefficients in each region with computational complexity that remains on the order of a traditional adaptive linear equalizer [8]. The resulting algorithm can therefore successfully navigate the short-data record regime, by placing more emphasis on lower-order models, while achieving the ultimate precision of higher order models as the data record grows to accommodate them. The introduced equalizer

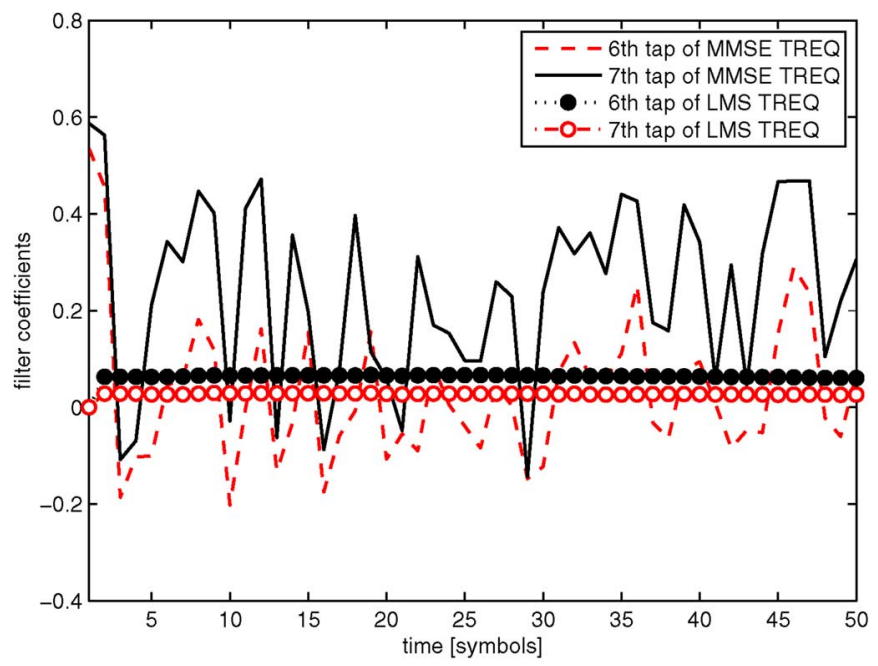

Fig. 1. An example of time varying filter coefficients of an MMSE turbo equalizer (TREQ) and steady state filter coefficients of an LMS turbo equalizer (TREQ) in a time invariant ISI channel $[0.227,0.46,0.688,0.46,0.227]$ at the second turbo iteration. $(S N R=10 \mathrm{~dB}$, feedforward filter length $=15$, step size $=0.001$, BPSK, random interleaver and $1 / 2$ rate convolutional code with constraint length of 3 are used).

can be shown to asymptotically (and uniformly) achieve the performance of the best piecewise linear equalizer that could have been constructed, given full knowledge of the channel and the received data sequence in advance. Furthermore, the mean square error (MSE) of this equalizer is shown to converge to that of the minimum MSE (MMSE) estimator (which is a nonlinear function of the soft information) as the depth of the context tree and data length increase.

Context trees and context tree weighting are extensively used in data compression [15], coding and data prediction [16]-[18]. In the context of source coding and universal probability assignment, the context tree weighting method is mainly used to calculate a weighted mixture of probabilities generated by the piecewise Markov models represented on the tree [15]. In nonlinear prediction, context trees are used to represent piecewise linear models by partitioning the space of past regressors [16], [18], specifically for labeling the past observations based on a certain criteria. Note that although we use the notion of context trees for nonlinear modeling as in [15], [17]-[20], our results and usage of context trees differ from [15], [17]-[19] in a number of important ways. The "context" used in our context trees correspond to a spatial parsing of the soft information space, rather than the temporal parsing as studied in [15], [17], [18].

In addition, the context trees here are specifically used to represent the nonlinear dependency of equalizer coefficients on the soft information. We emphasize that such application is naturally different than application of context trees to data prediction, where nonlinear prediction is carried out by employing context trees to partition space of past relatives past regressors, either for clean [19] or noisy [20] past observations. In this sense, as an example, the time adaptation here is mainly (in addition to learning) due to the time variation of the soft information coming from the decoder, unlike the time dependent learning in [20] or [19]. Hence, here, we explicitly calculate the MSE performance and quantify the difference between the MSE 
of the context tree algorithm and the MSE of the linear MMSE equalizer, which is the main objective.

The paper is organized as follows. In Section II, we introduce the basic system description and provide the objective of the paper. The nonlinear equalizers studied are introduced in Section III. In Section III, we first introduce a partitioned linear turbo equalization algorithm, where the partitioning of the regions is fixed. We continue in Section III-B with the turbo equalization framework using context trees, where the corresponding algorithm with the guaranteed performance bounds is introduced. Furthermore, we provide the MSE performance of all the algorithms introduced and compare them to the MSE performance of the linear MMSE equalizer. The paper concludes with numerical examples demonstrating the performance gains and the learning mechanism of the algorithm.

\section{SYSTEM DESCRIPTION}

Throughout the paper, all vectors are column vectors and represented by boldface lowercase letters. Matrices are represented by boldface uppercase letters. Given a vector $\boldsymbol{x},\|\boldsymbol{x}\|=\sqrt{\boldsymbol{x}^{H} \boldsymbol{x}}$ is the $l_{2}$-norm, where $\boldsymbol{x}^{H}$ is the conjugate transpose, $\boldsymbol{x}^{T}$ is the ordinary transpose and $\boldsymbol{x}^{*}$ is the complex conjugate. For a random variable $x$ (or a vector $\boldsymbol{x}$ ), $E[x]=\bar{x}($ or $E[\boldsymbol{x}]=\overline{\boldsymbol{x}}$ ) is the expectation. For a vector $\boldsymbol{x}, \operatorname{diag}(\boldsymbol{x})$ is a diagonal matrix constructed from the entries of $\boldsymbol{x}$ and $x(i)$ is the $i$ th entry of the vector. For a square matrix $\boldsymbol{M}, \operatorname{tr}(\boldsymbol{M})$ is the trace. The sequences are represented using curly brackets, e.g., $\{x(t)\} \cdot \bigcup_{i=1}^{N} A_{i}$ denotes the union of the sets $A_{i}$, where $i=1, \ldots, N$. The vec(.) operator stacks columns of a matrix of dimension $m \times n$ into an $m n \times 1$ column vector [21]. Furthermore, for functions $f(x)$ and $g(x)$, $f(x)=O(g(x))$ represents $\limsup _{x \rightarrow \infty} \frac{f(x)}{g(x)}<\infty$.

The block diagram of the system we consider with a linear turbo equalizer is shown in Fig. 2. The information bits $\{b(t)\}\{\hat{b}(t)\}$ are first encoded using an error correcting code (ECC) and then interleaved to generate $\{c(t)\}$. The interleaved code bits $\{c(t)\}$ are transmitted after symbol mapping, e.g., $x(t)=(-1)^{c(t)}$ for BPSK signaling, through a baseband discrete-time channel with a finite-length impulse response $\{h(t)\}, t=0,1, \ldots, M-1$, represented by $\boldsymbol{h} \triangleq[h(M-1), \ldots, h(0)]^{T}$. The communication channel $\boldsymbol{h}$ is unknown. The transmitted signal $\{x(t)\}$ is assumed to be uncorrelated due to the interleaver. The received signal $y(t)$ is given by

$$
y(t) \triangleq \sum_{k=0}^{M-1} h(k) x(t-k)+n(t)
$$

where $\{n(t)\}$ is the additive complex white Gaussian noise with zero mean and circular symmetric variance $\sigma_{n}^{2}$. If a linear equalizer is used to reduce the ISI, then the estimate of the desired data, i.e., $\hat{x}(t)$, using the received data $y(t)$ is given by

$$
\hat{x}(t)=\boldsymbol{w}^{T}(t)[\boldsymbol{y}(t)-\overline{\boldsymbol{y}}(t)]+\bar{x}(t),
$$

where $\boldsymbol{w}(t)=\left[w\left(t, N_{2}\right), \ldots, w\left(t,-N_{1}\right)\right]^{T}$ is length $N=N_{1}+$ $N_{2}+1$ linear equalizer, $\boldsymbol{y}(t) \triangleq\left[y\left(t-N_{2}\right), \ldots, y\left(t+N_{1}\right)\right]^{T}$ and note that we use negative indices with a slight abuse of notation. The received data vector $\boldsymbol{y}(t)$ is given by $\boldsymbol{y}(t)=\boldsymbol{H} \boldsymbol{x}(t)+\boldsymbol{n}(t)$, where $\boldsymbol{x}(t) \triangleq\left[x\left(t-M-N_{2}+1\right), \ldots, x\left(t+N_{1}\right)\right]^{T}$ and $\boldsymbol{H} \in$
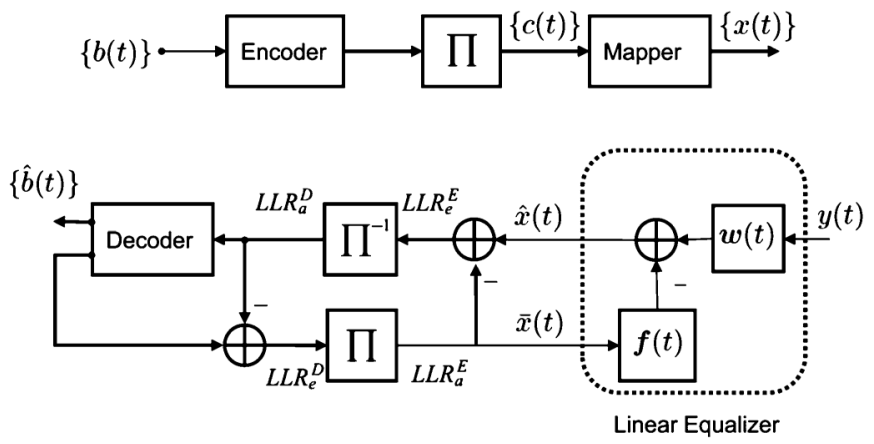

Fig. 2. Block diagram for a bit interleaved coded modulation transmitter and receiver with a linear turbo equalizer.

$\mathbb{C}^{N \times(N+M-1)}$ is the convolution matrix corresponding to $\boldsymbol{h}$, the estimate of $x(t)$ can be written as

$$
\hat{x}(t)=\boldsymbol{w}^{T}(t)[\boldsymbol{y}(t)-\boldsymbol{H} \overline{\boldsymbol{x}}(t)]+\bar{x}(t),
$$

given that the mean of the transmitted data is known.

However, in turbo equalization, instead of only using an equalizer, the equalization and the decoding are jointly performed iteratively at the receiver of Fig. 2. The equalizer computes the a posteriori information using the received signal, transmitted signal estimate, channel convolution matrix (if known) and a priori probability of the transmitted data. After subtracting the a priori information, $\operatorname{LLR}_{a}^{E}$, and de-interleaving the extrinsic information $\mathrm{LLR}_{e}^{E}$, a soft input soft output (SISO) channel decoder computes the extrinsic information $\mathrm{LLR}_{e}^{D}$ on coded bits, which are fed back to the linear equalizer as a priori information $\mathrm{LLR}_{a}^{E}$ after interleaving.

If one uses the linear MMSE equalizer in $\boldsymbol{w}(t)$, the mean and the variance of $x(t)$ are required to calculate $\boldsymbol{w}(t)$ and $\hat{x}(t)$. These quantities are computed using the a priori information from the decoder as $\bar{x}(t) \triangleq E\left[x(t):\left\{\operatorname{LLR}_{a}^{E}(t)\right\}\right]^{1}$ and $q(t) \triangleq E\left[x^{2}(t):\left\{\operatorname{LLR}_{a}^{E}(t)\right\}\right]-\bar{x}^{2}(t)$. As an example, for BPSK signaling, the mean and variance are given as $\bar{x}(t)=\tanh \left(\operatorname{LLR}_{a}^{E}(t) / 2\right)$ and $q(t)=1-|\bar{x}(t)|^{2}$. However, to remove dependency of $\hat{x}(t)$ to $\operatorname{LLR}_{a}^{E}(t)$ due to using $\bar{x}(t)$ and $q(t)$ in (1), one can set $\operatorname{LLR}_{a}^{E}(t)=0$ while computing $\hat{x}(t)$, yielding $\bar{x}(t)=0$ and $q(t)=1$ [5]. Then, the linear MMSE equalizer is given by

$$
\boldsymbol{w}(t)=\left[\boldsymbol{v}^{H}\left(\sigma_{n}^{2} \boldsymbol{I}+\boldsymbol{H}_{r} \boldsymbol{Q}(t) \boldsymbol{H}_{r}^{H}+\boldsymbol{v} \boldsymbol{v}^{H}\right)^{-1}\right]^{T},
$$

where $\boldsymbol{Q}(t)=E\left[(\boldsymbol{x}(t)-\overline{\boldsymbol{x}}(t))(\boldsymbol{x}(t)-\overline{\boldsymbol{x}}(t))^{H}:\left\{L L R_{a}^{E}(t)\right\}\right]$ is a diagonal matrix (due to uncorrelateness assumption on $x(t)$ ) with diagonal entries $\boldsymbol{Q}(t)=\operatorname{diag}(\boldsymbol{q}(t)), \boldsymbol{q}(t) \triangleq[q(t-M-$ $\left.\left.N_{2}+1\right), \ldots, q(t-1), q(t+1), \ldots, q\left(t+N_{1}\right)\right]^{T}, \boldsymbol{v} \in \mathbb{C}^{N}$ is the $\left(M+N_{2}\right)$ th column of $\boldsymbol{H}, \boldsymbol{H}_{r}$ is the reduced form of $\boldsymbol{H}$ where the $\left(M+N_{2}\right)$ th column is removed. The linear MMSE equalizer in (1) yields

$$
\begin{aligned}
\hat{x}(t) & =\boldsymbol{w}^{T}(t)[\boldsymbol{y}(t)-\boldsymbol{H} \overline{\boldsymbol{x}}(t)] \\
& =\boldsymbol{w}^{T}(t) \boldsymbol{y}(t)-\boldsymbol{f}^{T}(t) \overline{\boldsymbol{x}}(t),
\end{aligned}
$$

${ }^{1}$ With a slight abuse of notation, the expression $E[x(t): y(t)]$ is interpreted here and in the sequel as the expectation of $x(t)$ with respect to the prior distribution $y(t)$. 
where $\boldsymbol{f}(t) \triangleq \boldsymbol{H}^{T} \boldsymbol{w}(t)$. In this sense the linear MMSE equalizer can be decomposed into a feedforward filter $\boldsymbol{w}(t)$ processing $\boldsymbol{y}(t)$ and a feedback filter $\boldsymbol{f}(t)$ processing $\overline{\boldsymbol{x}}(t)$.

Remark 1: Both the linear MMSE feedforward and feedback filters are highly nonlinear functions of $\boldsymbol{q}$, i.e.,

$$
\begin{aligned}
\boldsymbol{w} & =\mathcal{W}(\boldsymbol{q}) \triangleq\left[\boldsymbol{v}^{H}\left(\sigma_{n}^{2} \boldsymbol{I}+\boldsymbol{H}_{r} \operatorname{diag}(\boldsymbol{q}) \boldsymbol{H}_{r}^{H}+\boldsymbol{v v}^{H}\right)^{-1}\right]^{T}, \\
\boldsymbol{f} & =\mathcal{F}(\boldsymbol{q}) \triangleq \boldsymbol{H}^{T} \mathcal{W}(\boldsymbol{q}),
\end{aligned}
$$

where $\mathcal{W}(\cdot), \mathcal{F}(\cdot): \mathbb{C}^{N+M-2} \rightarrow \mathbb{C}^{N}$. We point out that time variation in (2) is due to the time variation in the vector of variances $\boldsymbol{q}$ (assuming $\boldsymbol{h}$ is time-invariant).

To learn the corresponding feedforward and feedback filters that are highly nonlinear functions of $\boldsymbol{q}$, we use piecewise linear models based on vector quantization and context trees in the next section. The space spanned by $\boldsymbol{q}$ is partitioned into disjoint regions and a separate linear model is trained for each region to approximate functions $\mathcal{W}(\cdot)$ and $\mathcal{F}(\cdot)$ using piecewise linear models.

Note that if the channel is not known or estimated, one can directly train the corresponding equalizers in (3) using adaptive algorithms such as in [4], [12] without channel estimation or piecewise constant partitioning as done in this paper. In this case, one directly applies the adaptive algorithms to feedforward and feedback filters using the received data $\{y(t)\}$ and the mean vector $\overline{\boldsymbol{x}}(t)$ as feedback without considering the soft decisions as a priori probabilities. Assuming stationarity of $\overline{\boldsymbol{x}}(t)$, such an adaptive feedforward and feedback filters have Wiener solutions [12]

$$
\begin{aligned}
\boldsymbol{w} & =\left[\boldsymbol{v}^{H}\left(\sigma_{n}^{2} \boldsymbol{I}+\boldsymbol{H}_{r} E[\boldsymbol{Q}(t)] \boldsymbol{H}_{r}^{H}+\boldsymbol{v} \boldsymbol{v}^{H}\right)^{-1}\right]^{T}, \\
\boldsymbol{f} & =\boldsymbol{H}^{T} \boldsymbol{w} .
\end{aligned}
$$

Note that assuming stationarity of the log likelihood ratios [12], $E[\boldsymbol{Q}(t)]$ is constant in time, i.e., no time index for $\boldsymbol{w}, \boldsymbol{f}$ in (5). When PSK signaling is used such that $E\left[|x(t)|^{2}:\left\{\operatorname{LLR}_{a}^{E}(t)\right\}\right]=1$, the filter coefficient vector in (5) is equal to the coefficient vector of the MMSE equalizer in [5] with the time averaged soft information, i.e., time average instead of an ensemble mean. Comparing (5) and (2), we observe that using the time averaged soft information does not degrade equalizer performance in the no a priori information, i.e., $\boldsymbol{Q}(t)=\boldsymbol{I}$ or perfect $a$ priori information, i.e., $\boldsymbol{Q}(t)=\mathbf{0}$, cases. In addition, the performance degradation in moderate ISI channels is often small [5] when perfect channel knowledge is used. However, the performance gap increases in ISI channels that are more difficult to equalize, even in the high SNR region [12], since the effect of the filter time variation increases in the high SNR region. Comparison of an exact MMSE turbo equalizer without channel estimation error and an MMSE turbo equalizer with the time averaged soft variances (i.e. when an ideal filter for the converged adaptive turbo equalizer is used) via the EXIT chart [22] is given in Fig. 3. As the adaptive turbo equalizer, a decision directed (DD) LMS turbo equalizer is used in the data transmission period, while LMS is run on the received signals for the first turbo iteration and on the received signals and training symbols for the rest of turbo iterations in the training period. Note that the tentative decisions can be

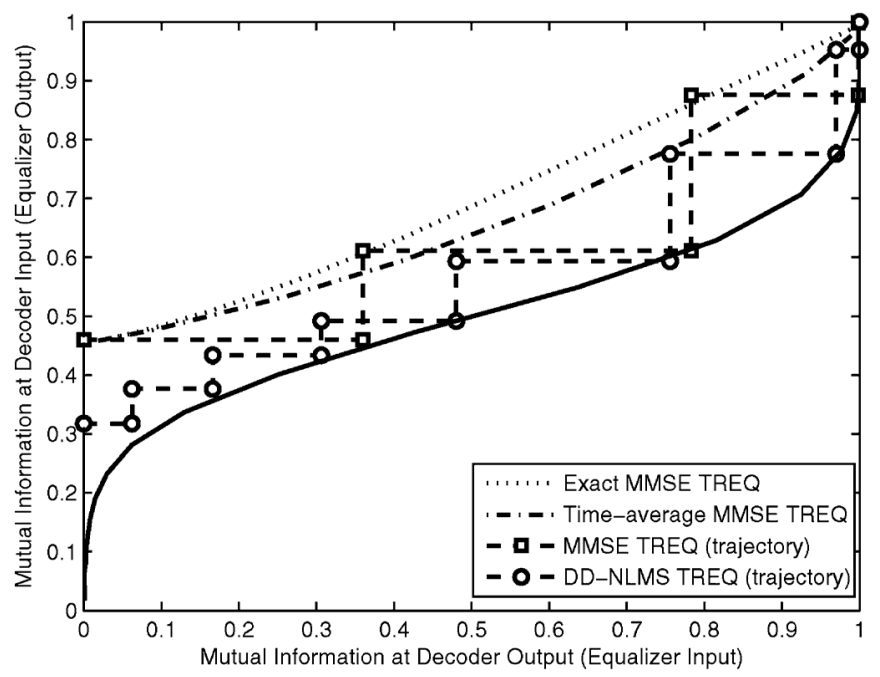

Fig. 3. The EXIT chart for the exact MMSE turbo equalizer, the LMS turbo equalizer and their trajectory in a time invariant ISI channel $[0.227,0.46,0.688,0.46,0.227]^{T}$. Here, we have SNR $=13 \mathrm{~dB}, N_{1}=9$, $N_{2}=5$, feedback filter length $N+M-1=19$, data block length $=8192$, training data length $=2048, \mu=0.001$, BPSK signaling, random interleaver and $\frac{1}{2}$ rate convolutional code with constraint length of 3 .

taken as the hard decisions at the output of the linear equalizer or as the soft decisions from the total LLRs at the output of decoder. When we consider nonideality, both of the MMSE turbo equalizer with channel estimation error and DD-LMS turbo equalizer loose mutual information at the equalizer in first few turbo iterations. ${ }^{2}$ Even though there is a loss in mutual information at the equalizer in the first and second turbo iteration due to using decision directed data or channel estimation error, both algorithms follow their ideal performance at the end. (i.e., the DD LMS turbo equalizer can achieve the performance of the time-average MMSE turbo equalizer as the decision data gets more reliable). However, there is still a gap in achieved mutual information between the exact MMSE turbo equalizer and the LMS adaptive turbo equalizer except for the no $a$ priori information and perfect a priori information cases. Note that such a gap can make an adaptive turbo equalizer become trapped at lower SNR region while an MMSE turbo equalizer converges as turbo iteration increases.

To remedy this, in the next section, we introduce piecewise linear equalizers to approximate $\mathcal{W}(\cdot)$ and $\mathcal{F}(\cdot)$. We first discuss adaptive piecewise linear equalizers with a fixed partition of $\mathbb{C}^{N+M-2}$ (where $\boldsymbol{q} \in \mathbb{C}^{N+M-2}$ ). Then, we introduce adaptive piecewise linear equalizers using context trees that can learn the best partition from a large class of possible partitions of $\mathbb{C}^{N+M-2}$.

\section{NONLINEAR TURbo EQUaLIZATION USING PIECEWISE LINEAR MODELS}

\section{A. Piecewise Linear Turbo Equalization with Fixed Partitioning}

In this section, we divide the space spanned by $\boldsymbol{q} \in$ $[0,1]^{N+M-2}$ (assuming BPSK signaling for notational simplicity) into disjoint regions $V_{k}$, e.g., $[0,1]^{N+M-2}=\bigcup_{k=1}^{K} V_{k}$

\footnotetext{
${ }^{2}$ This performance loss in the first few turbo iterations can cause the iterative process to terminate prematurely in low SNR region.
} 
for some $K$ and train an independent linear equalizer in each region $V_{k}$ to yield a final piecewise linear equalizer to approximate $\boldsymbol{w}=\mathcal{W}(\boldsymbol{q})$ and $\boldsymbol{f}=\mathcal{F}(\boldsymbol{q})$. As an example, given $K$ such regions, suppose a time varying linear equalizer is assigned to each region as $\boldsymbol{w}_{k}(t), \boldsymbol{f}_{k}(t), k=1, \ldots, K$, such that at each time $t$, if $\boldsymbol{q}(t) \in V_{k}$, the estimate of the received signal is given as

$$
\begin{aligned}
\hat{x}_{k}(t) & \triangleq \boldsymbol{w}_{k}^{T}(t) \boldsymbol{y}(t)-\boldsymbol{f}_{k}^{T}(t) \overline{\boldsymbol{x}}(t) \\
\hat{x}(t) & =\hat{x}_{k}(t) .
\end{aligned}
$$

We emphasize that the time variations in $\boldsymbol{w}_{k}(t)$ and $\boldsymbol{f}_{k}(t)$ in (6) are not due to the time variation in $\boldsymbol{q}(t)$ unlike (3). The filters $\boldsymbol{w}_{k}(t)$ and $\boldsymbol{f}_{k}(t)$ are time varying since they are produced by adaptive algorithms sequentially learning the corresponding functions $\mathcal{W}(\cdot)$ and $\mathcal{F}(\cdot)$ in region $V_{k}$. Note that if $K$ is large and the regions are dense such that $\mathcal{W}(\boldsymbol{q})$ (and $\mathcal{F}(\boldsymbol{q})$ ) can be considered constant in $V_{k}$, say equal to $\mathcal{W}\left(\boldsymbol{q}_{k}\right)$ for some $\boldsymbol{q}_{k}$ in region $V_{k}$, then if the adaptation method used in each region converges successfully, this yields $\boldsymbol{w}_{k}(t) \rightarrow \mathcal{W}\left(\boldsymbol{q}_{k}\right)$ and $\boldsymbol{f}_{k}(t) \rightarrow \mathcal{F}\left(\boldsymbol{q}_{k}\right)$ as $t \rightarrow \infty$. Hence, if these regions are dense and there is enough data to learn the corresponding models in each region, then this piecewise model can approximate any smoothly varying $\mathcal{W}(\boldsymbol{q})$ and $\mathcal{F}(\boldsymbol{q})$ [23].

In order to choose the corresponding regions $V_{1}, \ldots, V_{K}$, we apply a vector quantization (VQ) algorithm to the sequence of $\{\boldsymbol{q}(t)\}$, such as the LBG VQ algorithm [24]. If a VQ algorithm with $K$ regions and Euclidean distance is used for clustering, then the centroids and the corresponding regions are defined as

$\tilde{\boldsymbol{q}}_{k} \triangleq \frac{\sum_{t, \boldsymbol{q}(t) \in V_{k}} \boldsymbol{q}(t)}{\sum_{t, \boldsymbol{q}(t) \in V_{k}} 1}$,

$V_{k} \triangleq\left\{\boldsymbol{q}:\left\|\boldsymbol{q}-\tilde{\boldsymbol{q}}_{k}\right\| \leq\left\|\boldsymbol{q}-\tilde{\boldsymbol{q}}_{i}\right\|, \quad i=1, \ldots, K, i \neq k\right\}$,

where $\boldsymbol{q}(t)=\left[q\left(t-M-N_{2}+1\right), \ldots, q(t-1), q(t+\right.$ $\left.1), \ldots, q\left(t+N_{1}\right)\right]^{T}$, and $\boldsymbol{q} \in \mathbb{C}^{N+M-2}$. We emphasize that we use a VQ algorithm on $\{\boldsymbol{q}(t)\}$ to construct the corresponding partitioned regions in order to concentrate on $\boldsymbol{q}$ vectors that are in $\{\boldsymbol{q}(t)\}$ since $\mathcal{W}(\cdot)$ and $\mathcal{F}(\cdot)$ should only be learned around $\boldsymbol{q} \in\{\boldsymbol{q}(t)\}$, not for all $\mathbb{C}^{N+M-2}$. After the regions are constructed using the VQ algorithm and the corresponding filters in each region are trained with an appropriate adaptive method, the estimate of $x(t)$ at each time $t$ is given as $\hat{x}(t)=\hat{x}_{i}(t)$ if $i=\arg \min _{k}\left\|\boldsymbol{q}(t)-\tilde{\boldsymbol{q}}_{k}\right\|$.

In Fig. 4, we introduce such a sequential piecewise linear equalizer that uses the LMS update to train its equalizer filters. Here, $\mu$ is the learning rate of the LMS updates. One can use different adaptive methods instead of the LMS update, such as the RLS or NLMS updates [25], by only changing the filter update steps in Fig. 4. The algorithm of Fig. 4 has access to training data of size $T$. After the training data is used, the adaptive methods work in decision directed mode [25]. Since there are no a priori probabilities in the first turbo iteration, this algorithm uses an LMS update to train a linear equalizer with only the feedforward filter, i.e., $\hat{x}(t)=\boldsymbol{w}^{T}(t) \boldsymbol{y}(t)$, without any regions or mean vectors. Note that an adaptive feedforward linear filter $\boldsymbol{w}(t)$ trained on only $\{y(t)\}$ without a priori probabilities (as in the first iteration) converges to [12] (assuming zero variance in convergence)

$$
\lim _{t \rightarrow \infty} \boldsymbol{w}(t)=\boldsymbol{w}_{o} \triangleq\left[\boldsymbol{v}^{H}\left(\sigma_{n}^{2} \boldsymbol{I}+\boldsymbol{H}_{r} \boldsymbol{H}_{r}^{H}+\boldsymbol{v} \boldsymbol{v}^{H}\right)^{-1}\right]^{T},
$$

which is the linear MMSE feedforward filter in (2) with $Q(t)=$ I.

In the pseudo-code in Fig. 4, the iteration numbers are displayed as superscripts, e.g., $\boldsymbol{w}_{i}^{(m)}(t), \boldsymbol{f}_{i}^{(m)}(t)$ are the feedforward and feedback filters for the $m$ th iteration corresponding to the $i$ th region, respectively. After the first iteration when $\{\boldsymbol{q}(t)\}$ become available, we apply the VQ algorithm to get the corresponding regions and the centroids. Then, for each region, we run a separate LMS update to train a linear equalizer and construct the estimated data as in (6). In the start of the second iteration, in line A, each feedforward filter is initialized by the feedforward filter trained in the first iteration. Furthermore, although the linear equalizers should have the form $\boldsymbol{w}_{k}^{T}(t) \boldsymbol{y}(t)-\boldsymbol{f}_{k}^{T}(t) \overline{\boldsymbol{x}}(t)$, since we have the correct $\boldsymbol{x}(t)$ in the training mode for $t=1, \ldots, T$, the algorithms are trained using $\boldsymbol{w}_{k}^{T}(t) \boldsymbol{y}(t)-\boldsymbol{f}_{k}^{T}(t)\left[\boldsymbol{I}-\operatorname{diag}\left(\tilde{\boldsymbol{q}}_{k}\right)\right]^{1 / 2} \boldsymbol{x}(t)$ in (line B), i.e., $\boldsymbol{x}(t)$ is scaled using $\left[\boldsymbol{I}-\operatorname{diag}\left(\tilde{\boldsymbol{q}}_{k}\right)\right]^{1 / 2}$, to incorporate the uncertainty during training [26]. After the second iteration, in the start of each iteration, in line $\mathrm{C}$, the linear equalizers in each region, say $k$, are initialized using the filters trained in the previous iteration that are closest to the $k$ th region, i.e., $j=\arg \min _{i} \| \tilde{\boldsymbol{q}}_{k}^{(m)}-$ $\tilde{\boldsymbol{q}}_{i}^{(m-1)} \|, \boldsymbol{w}_{k}^{(m)}(0)=\boldsymbol{w}_{j}^{(m-1)}(n)$, and $\boldsymbol{f}_{k}^{(m)}(0)=\boldsymbol{f}_{j}^{(m-1)}(n)$.

Assuming large $K$ with dense regions, we have $\boldsymbol{q}(t) \approx \tilde{\boldsymbol{q}}_{k}$ when $\boldsymbol{q}(t) \in V_{k}$. To get the vectors that the LMS trained linear filters in region $k$ eventually converge, i.e., the linear MMSE estimators assuming stationary $\overline{\boldsymbol{x}}$, we need to calculate $E[(\boldsymbol{x}(t)-$ $\left.\overline{\boldsymbol{x}}(t))(\boldsymbol{x}(t)-\overline{\boldsymbol{x}}(t))^{H}: \boldsymbol{q}(t)=\tilde{\boldsymbol{q}}_{k}\right]$, which is assumed to be diagonal due to the interleaving [12], yielding

$$
\begin{gathered}
E\left\{[\boldsymbol{x}(t)-\overline{\boldsymbol{x}}(t)][\boldsymbol{x}(t)-\overline{\boldsymbol{x}}(t)]^{H}: \boldsymbol{q}(t)=\tilde{\boldsymbol{q}}_{k}\right\} \\
=E\left\{E \left\{[\boldsymbol{x}(t)-\overline{\boldsymbol{x}}(t)][\boldsymbol{x}(t)-\overline{\boldsymbol{x}}(t)]^{H}:\right.\right. \\
\left.\left.\left\{\mathrm{LLR}_{a}^{E}(t)\right\}, \boldsymbol{q}(t)=\tilde{\boldsymbol{q}}_{k}\right\}: \boldsymbol{q}(t)=\tilde{\boldsymbol{q}}_{k}\right\} \\
=\operatorname{diag}\left(\tilde{\boldsymbol{q}}_{k}\right)
\end{gathered}
$$

due to the definition of $\boldsymbol{q}(t)$ and assuming stationary distribution on $\bar{x}(t)$. This yields that the linear filters in region $k$ converge to

$$
\begin{aligned}
& \lim _{t \rightarrow \infty} \boldsymbol{w}_{k}(t)=\boldsymbol{w}_{k, o} \triangleq\left[\boldsymbol{v}^{H}\left(\sigma_{n}^{2} \boldsymbol{I}+\boldsymbol{H}_{r} \tilde{\boldsymbol{Q}}_{k} \boldsymbol{H}_{r}^{H}+\boldsymbol{v v}^{H}\right)^{-1}\right]^{T}, \\
& \lim _{t \rightarrow \infty} \boldsymbol{f}_{k}(t)=\boldsymbol{f}_{k, o} \triangleq \boldsymbol{H}^{T} \boldsymbol{w}_{k, o},
\end{aligned}
$$

where $\tilde{\boldsymbol{Q}}_{k} \triangleq \operatorname{diag}\left(\tilde{\boldsymbol{q}}_{k}\right)$, assuming zero variance at convergence. Hence, at each time $t$, assuming convergence, the difference between the MSE of the equalizer in (9) and the MSE of the linear MMSE equalizer in (2) is given by

$$
\begin{aligned}
& \| \boldsymbol{w}_{k, o}^{T} \boldsymbol{H}_{r} \boldsymbol{Q}(t) \boldsymbol{H}_{r}^{H} \boldsymbol{w}_{k, o}^{*}+\sigma_{n}^{2} \boldsymbol{w}_{k, o}^{T} \boldsymbol{w}_{k, o}^{*} \\
& \quad-\left[1-\boldsymbol{v}^{H}\left(\sigma_{n}^{2} \boldsymbol{I}+\boldsymbol{H}_{r} \boldsymbol{Q}(t) \boldsymbol{H}_{r}^{H}+\boldsymbol{v v}^{H}\right)^{-1} \boldsymbol{v}\right] \| \\
& \leq O\left(\left\|\boldsymbol{q}(t)-\tilde{\boldsymbol{q}}_{k}\right\|^{2}\right),
\end{aligned}
$$

as shown in Appendix A. Due to (10) as the number of piecewise linear regions, i.e., $K$, increases and $\left\|\boldsymbol{q}(t)-\tilde{\boldsymbol{q}}_{k}\right\|$ approaches 0 , the MSE of the converged adaptive filter more accurately approximates the MSE of the linear MMSE equalizer.

Note that the algorithm in Fig. 4 uses the LBG VQ for clustering and $K$ separate piecewise linear equalizers, one for each 


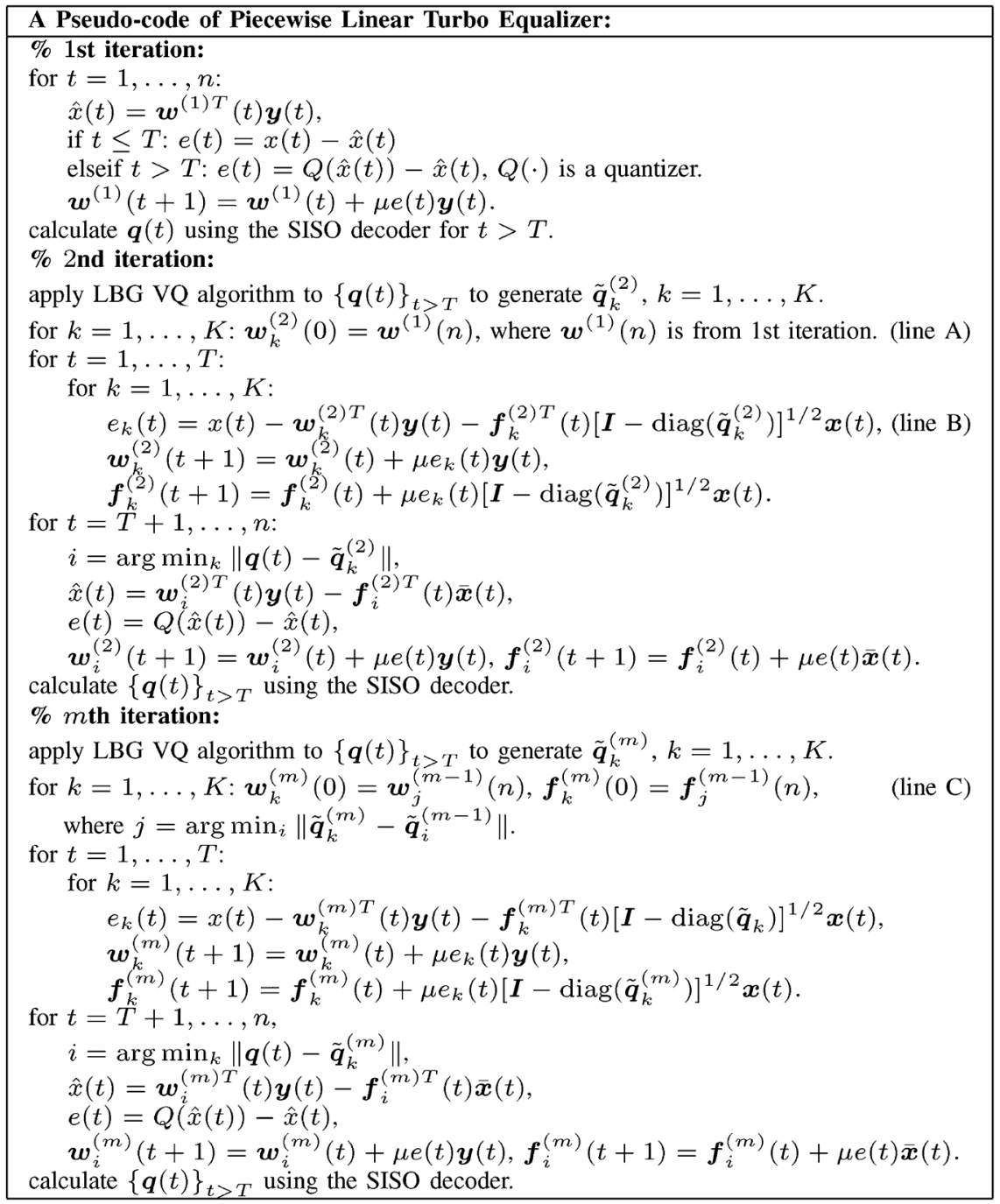

Fig. 4. A piecewise linear equalizer for turbo equalization. This algorithm requires $O(M+N)$ computations.

region. At the start of each turbo iteration, the LBG VQ algorithm requires $O(K L(N+M) \ln (n))$ computations [24], where $K$ is the number of clusters, $n$ is the data length, $N+M$ is the size of each variance vector and $L$ is an upper bound on the number of iterations that the LBG VQ requires for convergence. Then, at each time $t$, the $\mathrm{LBG} \mathrm{VQ}$ requires $O(K L(N+$ $M) \ln (n) / n)$ computations, i.e., $O(\ln (n) / n)$ number of additions and multiplications. ${ }^{3}$ At each time $t$, after observing $\boldsymbol{q}(t)$, the algorithm requires $O((M+N) \ln (K))$ computations to find the region that $\boldsymbol{q}(t)$ belongs to, $O((M+N))$ computations to calculate the output for that region and $O((M+N))$ computations to update the piecewise linear equalizer for that regions with the LMS algorithm. Hence, for each time $t$ (or per each output), the algorithm in Fig. 4 requires $O((M+N) \ln (K))+$ $O(K L(M+N) \ln (n) / n)$ computations.

In the algorithm of Fig. 4, the partition of the space of $q \in$ $[0,1]^{N+M-2}$ is fixed, i.e., partitioned regions are fixed at the start of the equalization, after the VQ algorithm, and we sequen-

\footnotetext{
${ }^{3}$ If the complexity of the LBG is significant compared to the adaptive algorithms, then one can replace it with a more appropriate partitioning method. As an example, the complexity of the LBG VQ can be significantly reduced by changing the distance measure to the Chaudhuris distance [27] instead of the Euclidian distance, which requires no multiplications.
}

tially learn a different linear equalizer for each region. Since the equalizers are sequentially learned with a limited amount of data, these may cause training problems if there is not enough data in each region. In other words, although one can increase $K$ to increase approximation power, if there is not enough data to learn the corresponding linear models in each region, this may deteriorate the performance. To alleviate this, one can try a piecewise linear model with smaller $K$ in the start of the learning and gradually increase $K$ to moderate values if enough data is available. In the next section, we examine the context tree weighting method that intrinsically does such weighting among different models based on their performance, hence, allowing the boundaries of the partition regions to be design parameters.

\section{B. Piecewise Linear Turbo Equalization Using Context Trees}

We first introduce a binary context tree to partition the side information space, $[0,1]^{N+M-2}$, into disjoint regions. An example binary context tree is provided in Fig. 5. In a binary context tree, starting from the root node, i.e., the top node, we have a left hand child and a right hand child. Each left hand child and right hand child have their own left hand and right hand children. This splitting yields a binary tree of depth $D$ with a total 


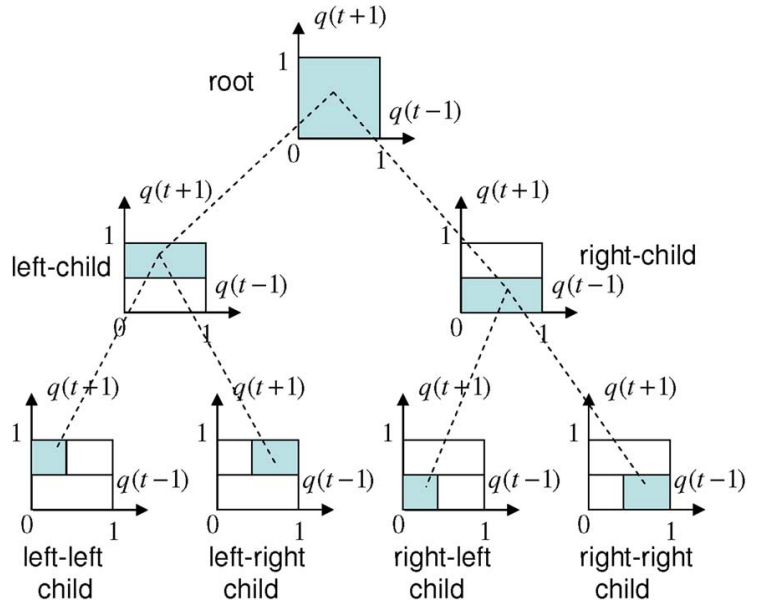

Fig. 5. A full binary context tree with depth, $D=2$, with 4 leaves. The leaves of this binary tree partitions $[0,1]^{2}$, i.e., $[q(t-1) q(t+1)] \in[0,1]^{2}$, into 4 disjoint regions.

of $2^{D}$ leaves at depth $D$ and a total of $2^{D+1}-1$ nodes including the root and the leaves. The example context tree in Fig. 5 has $D=2$ and partitions $[0,1]^{2}$, i.e., $\boldsymbol{q}=[q(t-1), q(t+1)]^{T} \in$ $[0,1]^{2}$, into 4 disjoint regions. Each one of these 4 disjoint regions is assigned to a leaf in this binary tree. Then, recursively, each internal node on this tree represents a region (shaded areas in Fig. 5), which is the union of the regions assigned to its children. Each node of the context tree represents a certain region (or part) of the side information space.

Using a binary context tree of depth $D$, one can represent a doubly exponential number, $m \approx 1.5^{2^{D}}$, of different "partitions" of the side information space. As an example, in Fig. 6, we provide the five partitions that can be represented by our binary tree with $D=2$ labeled as $\Gamma_{1}, \ldots, \Gamma_{5}$. Each partition represented by this context tree is assigned to a "complete" subtree. A complete subtree is constructed from a subset of the nodes of the original tree, starting from the same root node, and the union of the regions assigned to the leaves of the subtree yields $[0,1]^{N+M-2}$. For example, the subtree, $\Gamma_{3}$ in Fig. 6 , which has the left-child, the right-left child and the right-right child as its leaves provides a complete partition of $[0,1]^{N+M-2}$. For any subtree $i$ defined in the binary tree, if the regions assigned to its leaves are labeled as $V_{1, i}, \ldots, V_{K_{i}, i}$ where $K_{i}$ is the number of leaves of the subtree $i$, then $[0,1]^{N+M-2}=\bigcup_{k=1}^{K_{i}} V_{k, i}$. Each $V_{k, i}$ of the subtree corresponds to a node in the original tree.

To construct our context tree based piecewise linear equalizer, we first partition the side information space with the LBG VQ algorithm as in the previous section and then construct our context tree over these regions as follows. We emphasize that the context tree is not directly used to partition the side information space but rather represents the possible partitions that can be constructed as the union of the final regions produced by the LBG VQ. Suppose the LBG VQ algorithm is applied to $\{\boldsymbol{q}(t)\}$ with $K=2^{D}$ to generate $2^{D}$ regions [24]. These regions are assigned to the leaves of a binary context tree of depth $D$. Then, recursively, each internal node represents a region, which is the union of its children nodes. Note that one can arbitrarily assign the regions produced by the LBG VQ to the leaves of the context tree. However, the LBG VQ algorithm uses a tree notion similar to the context tree introduced in Fig. 6 such that the LBG VQ algorithm intrinsically constructs the context tree. The LBG VQ algorithm starts from a root node and calculates the mean of all the vectors in $\{\boldsymbol{q}(t)\}$ as the root codeword, and binary splits the data as well as the root codeword into two segments. Then, these newly constructed codewords are iteratively used as the initial codebook of the split segments. These two codewords are then split in four and the process is repeated until the desired number of regions are reached. At the end, this binary splitting and clustering yield $2^{D}$ regions with the corresponding centroids $\tilde{\boldsymbol{q}}_{i}, i=1, \ldots, 2^{D}$, which are assigned to the leaves of the context tree. Note that since each couple of the leaves (or nodes) come from a parent node after a binary splitting, these parent codewords are stored as the internal nodes of the context tree, i.e., the nodes that are generated by splitting a parent node are considered as siblings of this parent node where the centroid before splitting is stored. Hence, in this sense, the LBG VQ algorithm constructed the context tree.

Given such a context tree, we have $m \approx(1.5)^{2^{D}}$ different partitions of the space $[0,1]^{N+M-2}$ and can construct a piecewise linear equalizer, say $\hat{x}_{\Gamma_{i}}(t)$, as in Fig. 4 for each such partition. One of these partitions, with the piecewise adaptive linear model defined on it achieves the minimal loss, e.g., the minimal accumulated squared error $\min _{i} \sum_{t=1}^{n}\left(x(t)-\hat{x}_{\Gamma_{i}}(t)\right)^{2}$, for some $n$. However, the best piecewise model with the best partition is not known a priori. We point out that although we have a doubly exponential number of piecewise linear models defined on the context tree, all these piecewise linear equalizers are constructed using subsets of nodes $\rho \in\left\{1, \ldots, 2^{D+1}-1\right\}$ of the tree. Hence, suppose we number each node on this context tree $\rho=1, \ldots, 2^{D+1}-1$ and assign a linear equalizer to each node as $\hat{x}_{\rho}(t)=\boldsymbol{w}_{\rho}^{T}(t) \boldsymbol{y}(t)-\boldsymbol{f}_{\rho}^{T}(t) \overline{\boldsymbol{x}}(t)$. The linear models $\boldsymbol{w}_{\rho}(t), \boldsymbol{f}_{\rho}(t)$ that are assigned to node $\rho$, train only on the data assigned to that node as in Fig. 4, i.e., if $\boldsymbol{q}(t)$ is in the region that is assigned to the node $\rho$, say $V_{\rho}$, then $\boldsymbol{w}_{\rho}(t)$ and $\boldsymbol{f}_{\rho}(t)$ are updated. Then, the piecewise linear equalizer $\hat{x}_{\Gamma_{i}}(t)$ corresponding to any partition $\Gamma_{i}=\left\{V_{1, i}, \ldots, V_{K_{i}, i}\right\}$ is defined such that if $\boldsymbol{q}(t) \in V_{k, i}$ and $\rho$ is the node that is assigned to $V_{k, i}$, i.e., $V_{k, i}=V_{\rho}$, then

$$
\begin{aligned}
\hat{x}_{\Gamma_{i}}(t) & =\hat{x}_{\rho}(t) \\
& =\boldsymbol{w}_{\rho}^{T}(t) \boldsymbol{y}(t)-\boldsymbol{f}_{\rho}^{T}(t) \overline{\boldsymbol{x}}(t) .
\end{aligned}
$$

We emphasize that this observation is critical while we introduce an algorithm that achieves the performance of the best partition with the best linear model that achieves the minimal accumulated square-error with complexity only linear in the depth of the context tree per sample, i.e., complexity $O(D(N+M))$ instead of $O\left((1.5)^{2^{D}} D(N+M)\right)$, where $D$ is the depth of the tree.

Remark 2: We note that the partitioned model that corresponds to the union of the leaves, i.e., the finest partition, has the finest partition of the space of variances. Hence, it has the highest number of regions and parameters to model the nonlinear dependency. However, note that at each such region, the finest partition needs to train the corresponding linear equalizer that belongs to that region. As an example, the piecewise linear equalizer with the finest partition may not yield satisfactory results in the beginning of the adaptation if there is not enough data to train all the model parameters. In this sense, as will be 


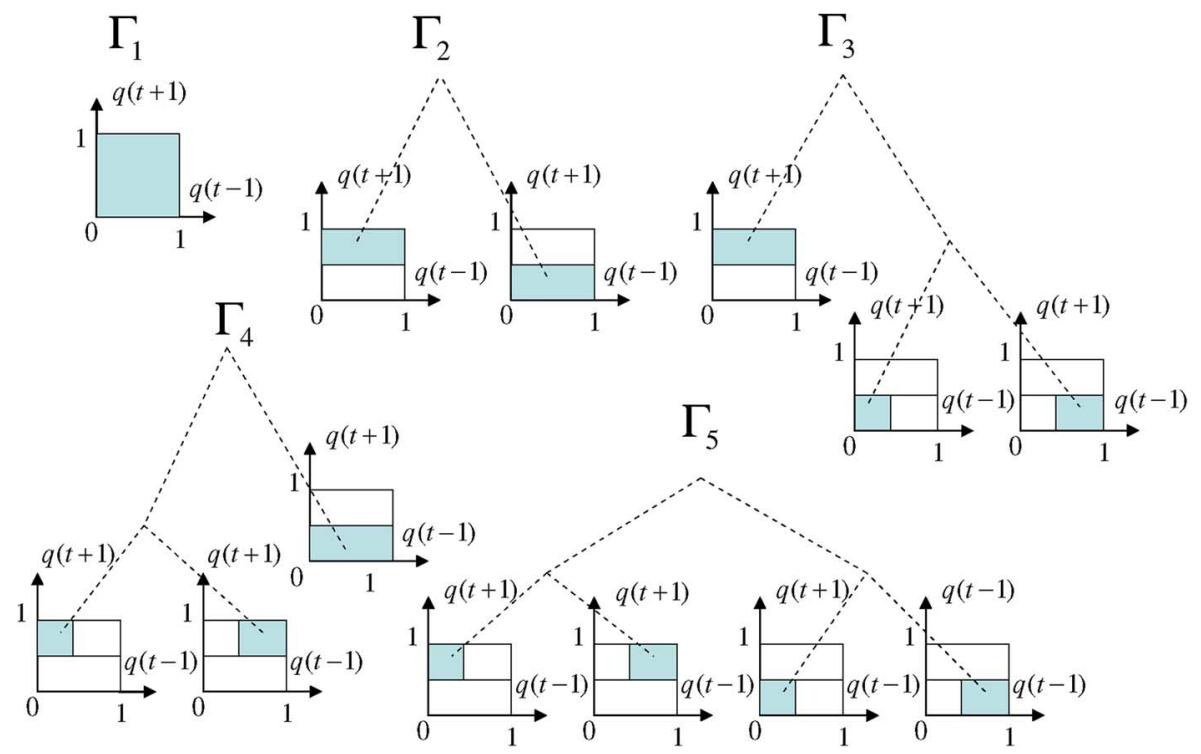

Fig. 6. All partitions of $[0,1]^{2}$ using binary context tree with $D=2$. Given any partition, the union of the regions represented by the leaves of each partition is equal to $[0,1]^{2}$.

shown, the context tree algorithm adaptively weights coarser and finer models based on their performance.

To accomplish this, we introduce the algorithm in Fig. 7, i.e., $\hat{x}_{\mathrm{ctw}}(t)$, that is constructed using the context tree weighting method introduced in [15]. The context tree based equalization algorithm implicitly constructs all $\hat{x}_{\Gamma_{i}}(t), i=1, \ldots, m$, piecewise linear equalizers and acts as if it had actually run all these equalizers in parallel on the received data. At each time $t$, the final estimation $\hat{x}_{\mathrm{ctw}}(t)$ is constructed as a weighted combination of all the outputs $\hat{x}_{\Gamma_{i}}(t)$ of these piecewise linear equalizers as

$$
\hat{x}_{\text {ctw }}(t)=\sum_{k=1}^{m} c_{k}(t) \hat{x}_{\Gamma_{k}}(t),
$$

where the combination weights are calculated proportional to the performance of each equalizer $\hat{x}_{\Gamma_{i}}(t)$ on the past data

$$
c_{k}(t) \triangleq \frac{2^{-C\left(\Gamma_{k}\right)} \exp \left\{-a \sum_{r=1}^{t-1}\left[x(r)-\hat{x}_{\Gamma_{k}}(r)\right]^{2}\right\}}{\sum_{j=1}^{m} 2^{-C\left(\Gamma_{j}\right)} \exp \left\{-a \sum_{r=1}^{t-1}\left[x(r)-\hat{x}_{\Gamma_{j}}(r)\right]^{2}\right\}},
$$

as explained in detail in Appendix B. However, as shown in (11) and explained in Appendix B, although there are $m$ different piecewise linear algorithms, at each time $t$, each $\hat{x}_{\Gamma_{i}}(t)$ is equal to one of the $D$ node estimations to which $\boldsymbol{q}(t)$ belongs. Hence, (12) can be implemented as a combination of only $D+1$ outputs as

$$
\hat{x}_{\mathrm{ctw}}(t)=\sum_{l=1}^{D+1} \beta_{k}(t) \hat{x}_{l(l)}(t),
$$

with certain combination weights $\beta_{k}(t)$ and nodes $l$. How the context tree algorithm keeps the track of these $m$ piecewise linear models as well as their performance-based combination weights with computational complexity only linear in the depth of the context tree is explained in Appendix B.

For the context tree algorithm, since there are no a priori probabilities in the first iteration, the first iteration of Fig. 7 is the same as the first iteration of Fig. 4. After the first iteration, to incorporate the uncertainty during training as in Fig. 4, the context tree algorithm is run by using weighted training data [26]. At each time $t>T, \hat{x}_{\mathrm{ctw}}(t)$ constructs its nonlinear estimation of $x(t)$ as follows. We first find the regions to which $\boldsymbol{q}(t)$ belongs. Due to the tree structure, one needs only find the leaf node in which $\boldsymbol{q}(t)$ lies and collect all the parent nodes towards the root node. The nodes to which $\boldsymbol{q}(t)$ belongs are stored in $\boldsymbol{l}$ in Fig. 7. The final estimate $\hat{x}_{\mathrm{ctw}}(t)$ is constructed as a weighted combination of the estimates generated in these nodes, i.e., $\hat{x}_{\rho}(t)$, $\rho \in \boldsymbol{l}$, where the weights are functions of the performance of the node estimates in previous samples.

For the algorithm in Fig. 7, at the start of each turbo iteration, we need to perform LBG VQ clustering, which requires $O\left(2^{D} L(N+M) \ln (n)\right) / n$ computations, i.e., additions and multiplications, for each time $t$. For each time $t$, we first need to find the nodes where $\boldsymbol{q}(t)$ belongs to, which requires $O\left(\ln \left(2^{D}\right)\right)$ computations (since due to the tree structure finding the leaf where $\boldsymbol{q}(t)$ belongs to is enough). Then, we need to perform $O(D(M+N))$ computations to calculate and combine the outputs of each node equalizer and require $O(D(M+N))$ computations to update the piecewise linear equalizers at these nodes. Hence, for each time $t$ (or per each output), the algorithm in Fig. 7 requires $O\left(2^{D} L(N+M) \ln (n)\right) / n+O(D(M+N))$ computations.

Theorem 2: Let $\{x(t)\},\{n(t)\}$ and $\{y(t)\}$ represent the transmitted, noise and received signals and $\{\boldsymbol{q}(t)\}$ represents the sequence of variances constructed using the a priori probabilities for each constellation point produced by the SISO decoder. Let $\hat{x}_{\rho}(t), \rho=1, \ldots, 2^{D+1}-1$, are estimates of $x(t)$ produced by the equalizers assigned to each node on the context tree. The algorithm $\hat{x}_{\mathrm{ctw}}(t)$, when applied to $\{y(t)\}$, for all $n$ achieves

$$
\sum_{t=1}^{n}\left(x(t)-\hat{x}_{\mathrm{ctw}}(t)\right)^{2} \leq \min _{\Gamma_{i}}\left\{\sum_{t=1}^{n}\left[x(t)-\hat{x}_{\Gamma_{i}}(t)\right]^{2}+2 K_{i}-1\right\}
$$

for all $i, i=1, \ldots, m \approx(1.5)^{2^{D}}$, assuming perfect feedback in decision directed mode i.e., $Q(\hat{x}(t))=x(t)$ when $t \geq T$, where $\hat{x}_{\Gamma_{i}}(t)$ is the equalizer constructed as

$$
\hat{x}_{\Gamma_{i}}(t)=\hat{x}_{\rho}(t),
$$


$\rho$ is the node assigned to the volume in $\Gamma_{i}=\left\{V_{1, i}, \ldots, V_{K_{i}, i}\right\}$ such that $\boldsymbol{q}(t)$ belongs and $K_{i}$ is the number of regions in $\Gamma_{i}$. If the estimation algorithms assigned to each node are selected as adaptive linear equalizers such as an RLS update based algorithm, (13) yields

$$
\begin{aligned}
& \sum_{t=1}^{n}\left[x(t)-\hat{x}_{\mathrm{ctw}}(t)\right]^{2} \\
& \leq \min _{i}\left\{\min _{\boldsymbol{w}_{k, i} \in \mathbb{C}_{\substack{, \boldsymbol{f}_{k, i} \in \mathbb{C}^{N+M-1} \\
k=1, \ldots, K_{i}}}}\right. \\
& \times \sum_{t=1}^{n}\left[x(t)-\boldsymbol{w}_{s_{i}(t), i}^{T} \boldsymbol{y}(t)-\boldsymbol{f}_{s_{i}(t), i}^{T} \bar{x}(t)\right]^{2} \\
& \left.+O((N+M) \ln (n))+2 K_{i}-1\right\} \text {. }
\end{aligned}
$$

where $s_{i}(t)$ is an indicator variable for $\Gamma_{i}$ such that if $\boldsymbol{q}(t) \in$ $V_{k, i}$, then $\left.s_{i}(t)=k \cdot\right\}$

An outline of the proof of this theorem is given in Appendix B.

Remark 3: We observe from (13) that the context tree algorithm achieves the performance of the best sequential algorithm among a doubly exponential number of possible algorithms corresponding to all partitions. Note that the bound in (13) holds uniformly for all $i$, however the bound is the largest for the finest partition, i.e., for the piecewise linear equalizer constructed using the regions assigned to the leaves. We observe from (14) that the context tree algorithm also achieves the performance of even the best piecewise linear model, independently optimized in each region, for all $i$ when the node estimators in each regions are adaptive algorithms that achieve the minimum least square-error.

\section{MSE Performance of the Context Tree Equalizer}

To get the MSE performance of the context tree equalizer, we observe that the result (14) in the theorem is uniformly true for any sequence $\{x(t)\}$. Hence, as a corollary to the theorem, taking the expectation of both sides of (14) with respect to any distribution on $\{x(t)\}$ yields the following:

$$
\begin{aligned}
& \text { Corollary: } \\
& \sum_{t=1}^{n} E\left\{\left[x(t)-\hat{x}_{\mathrm{ctw}}(t)\right]^{2}\right\}
\end{aligned}
$$

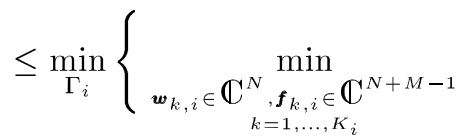

$$
\begin{aligned}
& \times \sum_{t=1}^{n} E\left\{\left[x(t)-\boldsymbol{w}_{s_{i}(t), i}^{T} \boldsymbol{y}(t)-\boldsymbol{f}_{s_{i}(t-1), i}^{T} \overline{\boldsymbol{x}}(t)\right]^{2}\right\} \\
& \left.+O((N+M) \ln (n))+2 K_{i}-1\right\} \text {. }
\end{aligned}
$$

Equation (14) is true for all $i$, and given $i$ for any $\boldsymbol{w}_{k, i}, \boldsymbol{f}_{k, i}$, $i=1, \ldots, K_{i}$, i.e.,

$$
\begin{aligned}
& \sum_{t=1}^{n}\left[x(t)-\hat{x}_{\mathrm{ctw}}(t)\right]^{2} \\
& \leq \sum_{t=1}^{n}\left[x(t)-\boldsymbol{w}_{s_{i}(t), i}^{T} \boldsymbol{y}(t)-\boldsymbol{f}_{s_{i}(t-1), i}^{T} \overline{\boldsymbol{x}}(t)\right]^{2} \\
& \quad+O((N+M) \ln (n))+2 K_{i}-1
\end{aligned}
$$

since (14) is true for the minimizing $i$ and equalizer vectors. Taking the expectation of both sides of (16) and minimizing with respect to $i$ and $\boldsymbol{w}_{k, i}, \boldsymbol{f}_{k, i}, i=1, \ldots, K_{i}$ yields the corollary.

We emphasize that the minimizer vectors $\boldsymbol{w}_{k, i}$ and $\boldsymbol{f}_{k, i}$ at the right hand side of (15) minimize the sum of all the MSEs. Hence, the corollary does not relate the MSE performance of the CTW equalizer to the MSE performance of the linear MMSE equalizer given in (2). However, if we assume that the adaptive filters trained at each node converge to their optimal coefficient vectors with zero variance and for sufficiently large $D$ and $n$, we have piecewise linear models such as for the finest partition

$$
\begin{aligned}
& \sum_{t=1}^{n}\left(x(t)-\hat{x}_{\Gamma_{|K|}}(t)\right)^{2} \\
& \approx \sum_{t=1}^{n}\left\{\left[x(t)-\boldsymbol{w}_{s_{|K|}(t),|K|, o}^{T} \boldsymbol{y}(t)-\boldsymbol{f}_{s_{\mid K}(t),|K|, o}^{T} \overline{\boldsymbol{x}}(t)\right]^{2}\right\},
\end{aligned}
$$

where we assumed that, for notational simplicity, the $|K|$ th partition is the finest partition, $\boldsymbol{w}_{\boldsymbol{s}_{|K|}(t),|K|, o}$ and $\boldsymbol{f}_{\boldsymbol{s}_{|K|}(t),|K|, o}$ are the MSE optimal filters (if defined) corresponding to the regions assigned to the leaves of the context tree. Note that we require $D$ to be large such that we can assume $\boldsymbol{q}(t)$ to be constant in each region such that these MSE optimal filters are well-defined. Since (14) is correct for all partitions and for the minimizing $\boldsymbol{w}, \boldsymbol{f}$ vectors, (14) holds for any $\boldsymbol{w}$ and $\boldsymbol{f}$ 's pairs including $\boldsymbol{w}_{s_{|K|}(t),|K|, o}$ and $\boldsymbol{f}_{s_{|K|}(t),|K|, o}$ pair. Since (14) in the theorem is uniformly true, taking the expectation preserves the bound and using (17), we have

$$
\begin{aligned}
& \frac{1}{n} \sum_{t=1}^{n} E\left\{\left[x(t)-\hat{x}_{\mathrm{ctw}}(t)\right]^{2}\right\} \\
& \leq \frac{1}{n} \sum_{t=1}^{n} E\left\{\left[x(t)-\boldsymbol{w}_{s_{|K|}(t),|K|, o}^{T} \boldsymbol{y}(t)\right.\right. \\
& \left.\left.-\boldsymbol{f}_{s_{\mid K}(t),|K|, o}^{T} \overline{\boldsymbol{x}}(t)\right]^{2}\right\}+O\left(\frac{2^{D+1}}{n}\right)
\end{aligned}
$$

since for the finest partition $K_{|K|}=2^{D}$. Using the MSE definition for each node in (18) yields

$$
\begin{aligned}
& \frac{1}{n} \sum_{t=1}^{n} E\left\{\left[x(t)-\hat{x}_{\mathrm{ctw}}(t)\right]^{2}\right\} \\
& \leq \frac{1}{n} \sum_{t=1}^{n}\left\{\boldsymbol{w}_{s_{|K|}^{T}(t), \mid K, o}^{T} \boldsymbol{H}_{r} \boldsymbol{Q}(t) \boldsymbol{H}_{r}^{H} \boldsymbol{w}_{s_{|K|}(t),|K|, o}^{*}\right.
\end{aligned}
$$

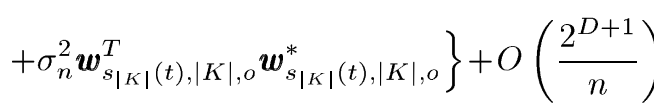

$$
\begin{aligned}
& \leq \frac{1}{n} \sum_{t=1}^{n}\left\{\min _{\boldsymbol{w}, \boldsymbol{f}} E\left\{\left[x(t)-\boldsymbol{w}^{T} \boldsymbol{y}(t)-\boldsymbol{f}^{T} \overline{\boldsymbol{x}}(t)\right]^{2} \mid \boldsymbol{q}(t)\right\}\right. \\
& \left.+O\left(\frac{1}{2^{D}}\right)\right\}+O\left(\frac{2^{D+1}}{n}\right),
\end{aligned}
$$

where (20) follows from assuming large $D$, the MSE in each node is bounded as in (10), i.e., $\| \boldsymbol{w}_{k, o}^{T} \boldsymbol{H}_{r} \boldsymbol{Q}(t) \boldsymbol{H}_{r}^{H} \boldsymbol{w}_{k, o}^{*}+$ $\sigma_{n}^{2} \boldsymbol{w}_{k, o}^{T} \boldsymbol{w}_{k, o}^{*}-\left[1-\boldsymbol{v}^{H}\left(\sigma_{n}^{2} \boldsymbol{I}+\boldsymbol{H}_{r} \boldsymbol{Q}(t) \boldsymbol{H}_{r}^{H}+\boldsymbol{v} \boldsymbol{v}^{H}\right)^{-1} \boldsymbol{v}\right] \| \leq$ $O\left(\left\|\boldsymbol{q}(t)-\tilde{\boldsymbol{q}}_{k}\right\|^{2}\right)$. Note that $O\left(\left\|\boldsymbol{q}(t)-\tilde{\boldsymbol{q}}_{k}\right\|\right)$ at the right hand side of (10) can further be upper bounded by $O\left(\frac{1}{2^{D}}\right)$ assuming 


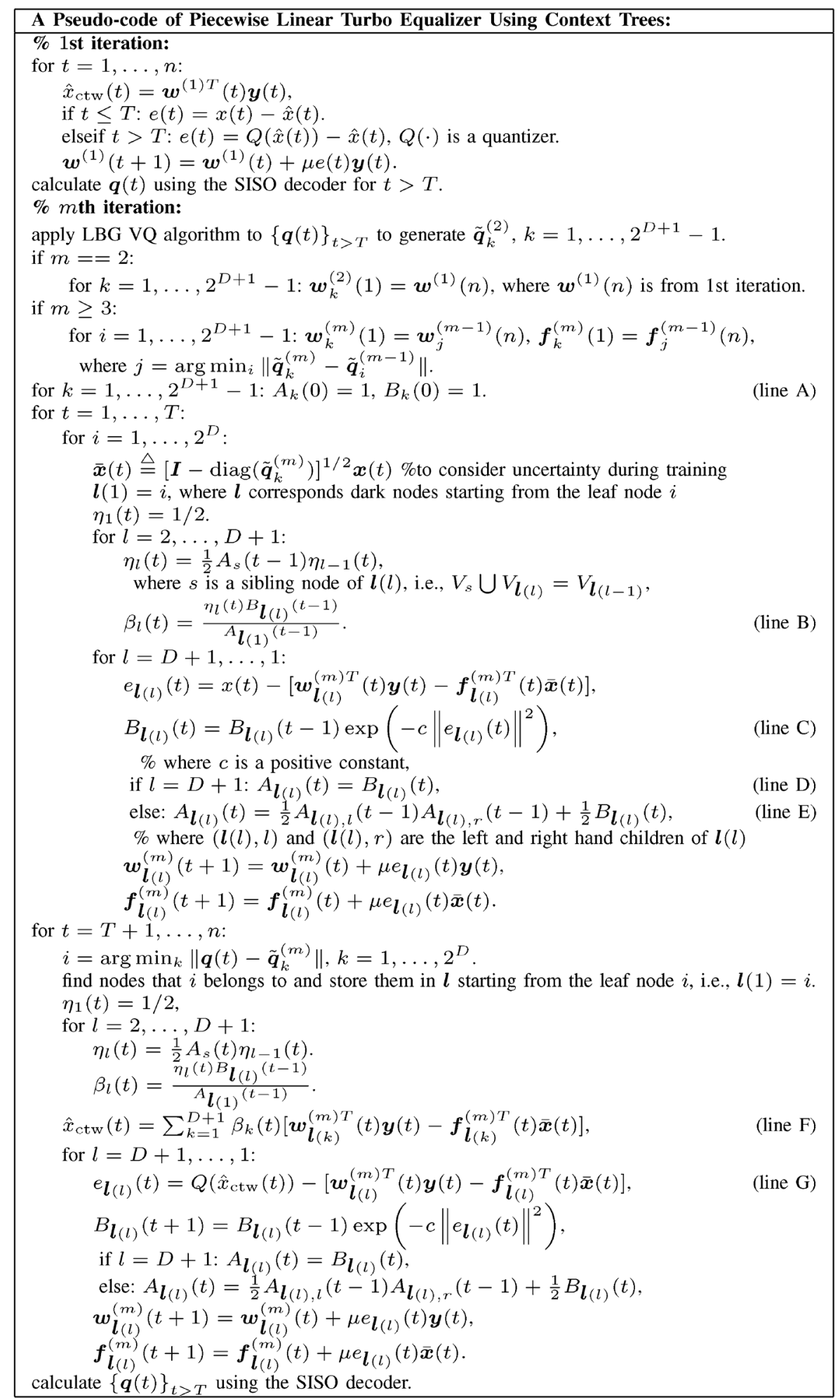

Fig. 7. A context tree based turbo equalization. This algorithm requires $O(D(M+N))$ computations.

large enough $D$ with the partition given in Fig. 5 since we have $2^{D}$ regions and $\|\boldsymbol{q}(t)\| \leq \sqrt{N+M-2}$. Hence, as $D \rightarrow \infty$, the context tree algorithm asymptotically achieves the performance of the linear MMSE equalizer, i.e., the equalizer that is nonlinear in the variances of the soft information. However, for this to happen, the sample length $n$ should go faster to infinity than $D$ as seen from the last term in (20).

\section{NUMERICAL EXAMPLES}

In the following, we simulate the performance of our algorithms under different scenarios. A rate one half convolutional code $^{4}$ with constraint length 3 and random interleaving is used.

\footnotetext{
${ }^{4} \mathrm{~A}$ recursive systematic convolutional code with a generator matrix $[101 ; 111]$ is used and Log-MAP decoding algorithm is considered as a decoding algorithm in this paper.
} 


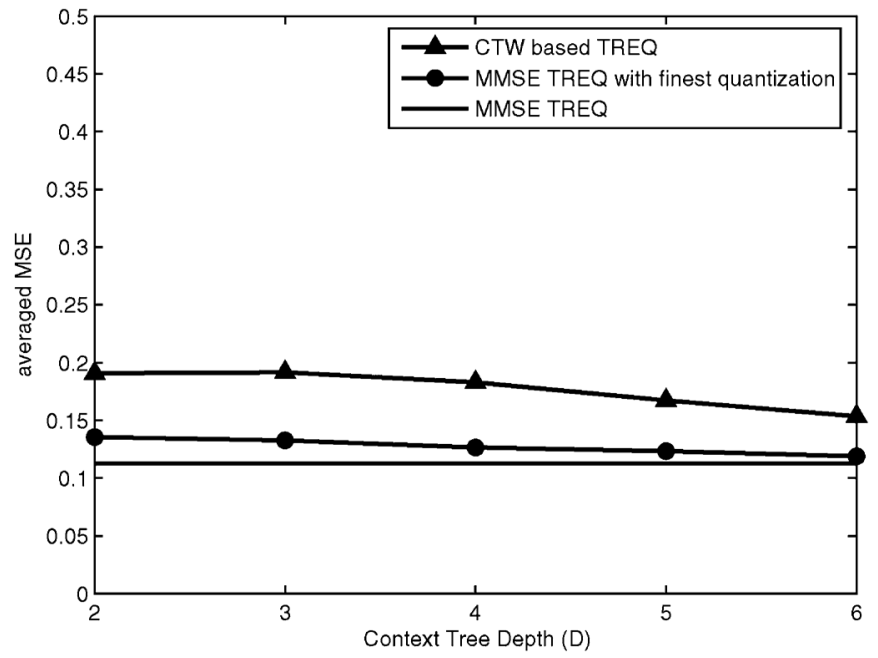

Fig. 8. Ensemble averaged MSE for the CTW equalizer over 5 turbo iterations at $12 \mathrm{~dB} \mathrm{~Eb} / \mathrm{N} 0$.

In the first set of experiments, we use the time invariant channels from [8] (Chapter 10)

$$
\begin{aligned}
\boldsymbol{h}_{1} & =[0.227,0.46,0.688,0.46,0.227]^{T} \\
\boldsymbol{h}_{2} & =[0.407,0.815,0.407]^{T}
\end{aligned}
$$

with the training size $T=1024$ and data length 5120 (excluding training). Training symbols are modulated with same modulation order of data symbols without encoding. We use two different adaptive algorithms including the LMS algorithm and the normalized LMS algorithm (NLMS) [28]. The decision directed mode is used for all the adaptive algorithms, e.g., for the ordinary LMS turbo equalizer we compete against and for all the node filters on the context tree. Our calculation of the extrinsic LLR at the output of the ordinary LMS algorithm is based on [10]. For all adaptive filters, we use $N_{1}=9, N_{2}=5$, length $N+M-1=19$ feedback filter. The learning rates for the LMS and the NLMS algorithms are set to $\mu=0.001$ and $\mu=0.05$, respectively. These learning rates are selected to guarantee the convergence of the ordinary LMS and NLMS filter in the training part. The same learning rate is used directly on the context tree without tuning for fair comparison.

As shown in Fig. 8, the MSE of the CTW equalizer decreases as the depth of the CTW increases as shown in (10). We also increase the data length as $5120 \times 2^{(D-1)}$, since the MSE of the CTW equalizer depends on both the depth of the context tree and the data length as demonstrated in (20). Furthermore, the MSEs of the linear MMSE TREQ and the linear MMSE TREQ with the finest partitioning are plotted as references, where the ideal channel estimation is assumed. Note that the difference between the MSE of the linear TREQ constructed using the finest partitioning and the MSE of the linear MMSE TREQ is bounded by the quantization error as shown in (10), where convergence of the linear filters for the finest partitioning is assumed. For the converged linear TREQ with the finest partitioning, we use the linear MMSE TREQ with the finest partition as the genie aided method. Note that, as expected, the MSE of the linear MMSE TREQ with the finest partition also decreases as the CTW depth increases since the quantization error decreases as the depth increases, i.e., the number of regions in the finest partition increases, where quantization level is given by $2^{D}$.

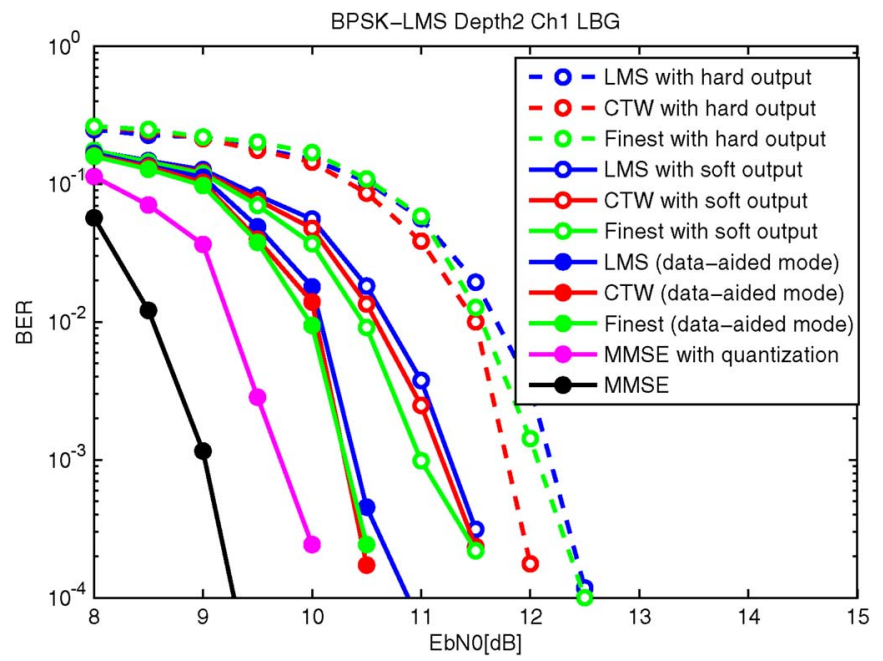

Fig. 9. BERs for an ordinary DD LMS algorithm, a CTW equalizer with $D=$ 2 and tree given in Fig. 5, the piecewise equalizer with the finest partition, i.e., $\hat{x}_{\Gamma_{5}}(t)$. (BPSK at the 7 th turbo iteration in the ISI channel, $\boldsymbol{h}_{1}$ ).

In Fig. 9, we plot BERs for an ordinary LMS algorithm, a context-tree equalization algorithm with $D=2$ given in Fig. 7 and the piecewise linear equalization algorithm with the finest partition, i.e., $\hat{x}_{\Gamma_{|K|}}(t)$, on the same tree. In a decision directed mode, hard decision data, soft decision data are used in these simulations. Data-aided adaptive algorithms are also demonstrated to show the ideal performance of the adaptive algorithms. As a reference, BERs of the MMSE TREQ and the MMSE TREQ with finest partition, where we assume that channel information is perfectly known at the receiver side. Note that the piecewise linear equalizer with the finest partition, i.e., $\Gamma_{5}$, in Fig. 6 , has the finest partition with the highest number of linear models, i.e., $2^{D}$ independent filters, for equalization. However, we emphasize that all the linear filters in the leaves should be sequentially trained for the finest partition. Hence, as explained in Section III-B, the piecewise linear model with the finest partition may yield inferior performance compared to the CTW algorithm that adaptively weights all the models based on their performance. We observe that the context tree equalizer outperforms the ordinary LMS equalizer and the equalizer corresponding to the finest partition in the hard output result.

In Fig. 10, we plot the weight evaluation of the context tree algorithm, i.e., the combined weight in line F of Fig. 7, to show the convergence of the CTW algorithm. Note that the combined weight vector for the CTW algorithm is only defined over the data length period 5120 at each turbo iteration, i.e., the combined weight vector is not defined in the training period. We collect the combined weight vectors for the CTW algorithm in the data period for all iterations and plot them in Fig. 10. This results in jumps in the figure, since at each discontinuity, i.e., after the data period, we switch to the training period and continue to train the node filters. The context tree algorithm, unlike the finest partition model, adaptively weights different partitions in each level. To see this, in Fig. 11(a), we plot weights assigned to each level in a depth $D=2$ context tree. We also plot the time evaluation of the performance measures $A_{\rho}(t)$ in Fig. 11(b). We observe that the context tree algorithm, as expected, at the start of the equalization divides the weights fairly uniformly among the partitions or node equalizers. However, naturally, as the training size increases, when there is enough 


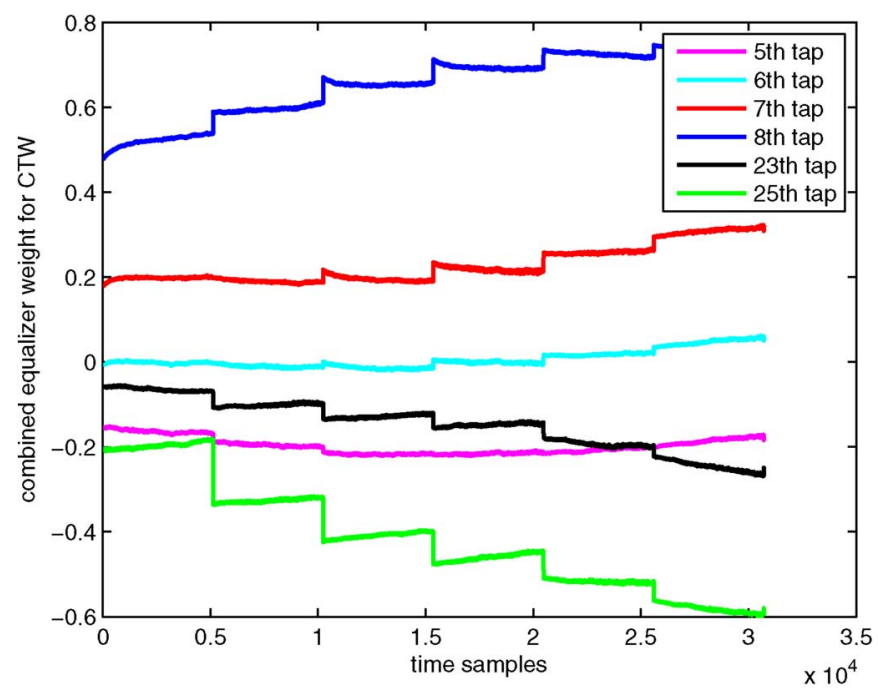

Fig. 10. Ensemble averaged combined weight vector for the CTW equalizer over 7 turbo iterations.

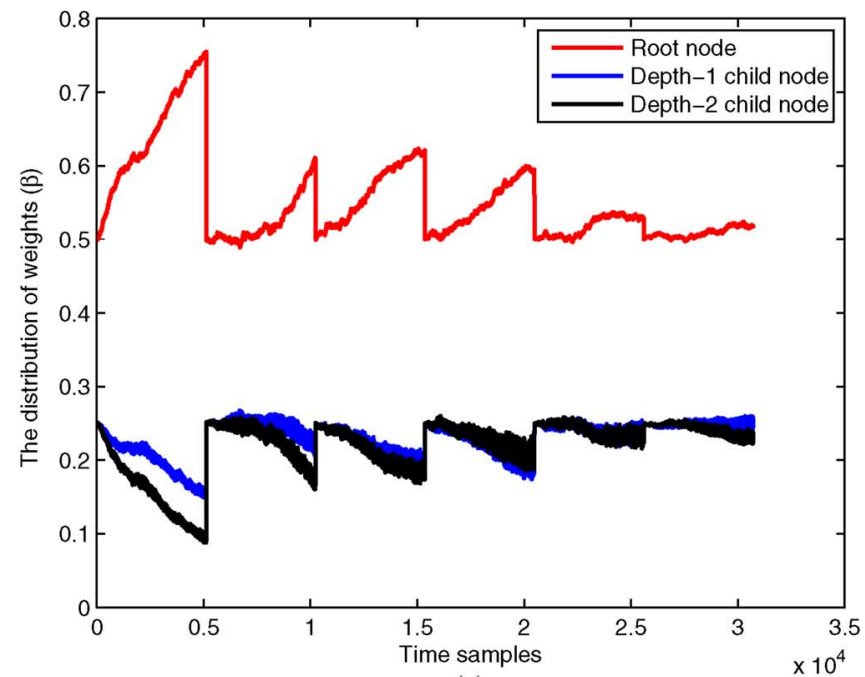

(a)

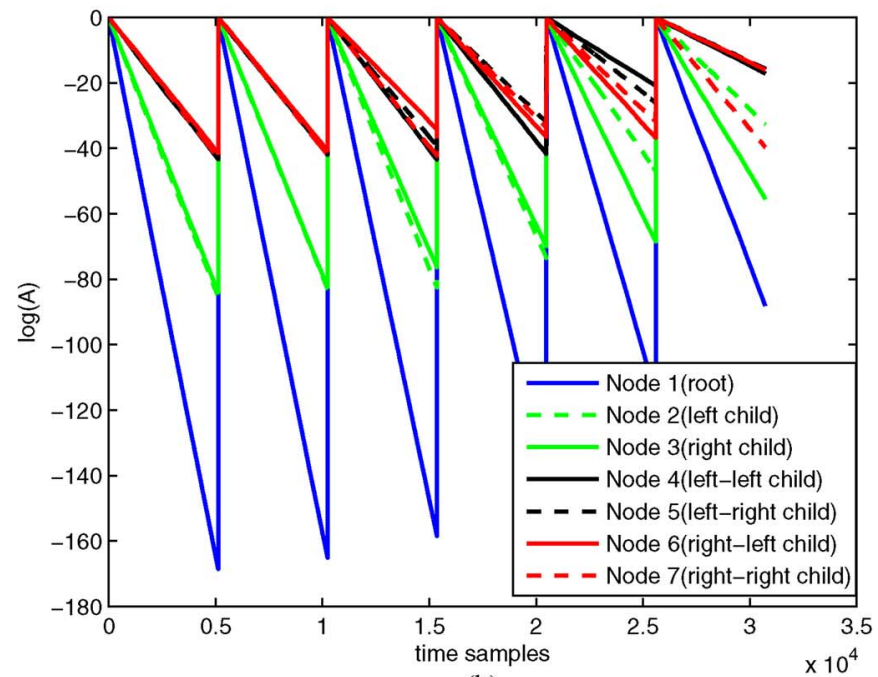

(b)

Fig. 11. (a) The distribution of the weights, i.e., values assigned to $\beta_{i}(t), i=$ $1,2,3$, such that $\beta_{i}(t)$ belongs to $i$ th level. (b) Time evaluation of $A_{\rho}(t)$ which represents the performance of the linear equalizer assigned to node $\rho$. Note that at each iteration, we reset $A_{\rho}(t)$ since a new tree is constructed using clustering.

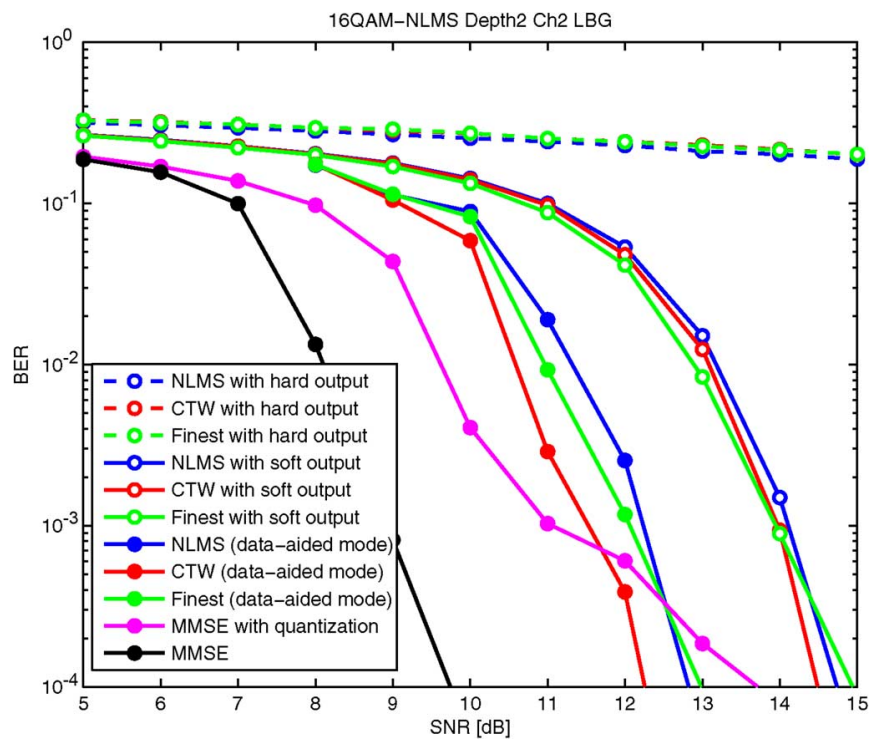

Fig. 12. BER comparison in the case of $16 \mathrm{QAM}$ at the 7 th turbo iteration in the ISI channel, $\boldsymbol{h}_{2}$.

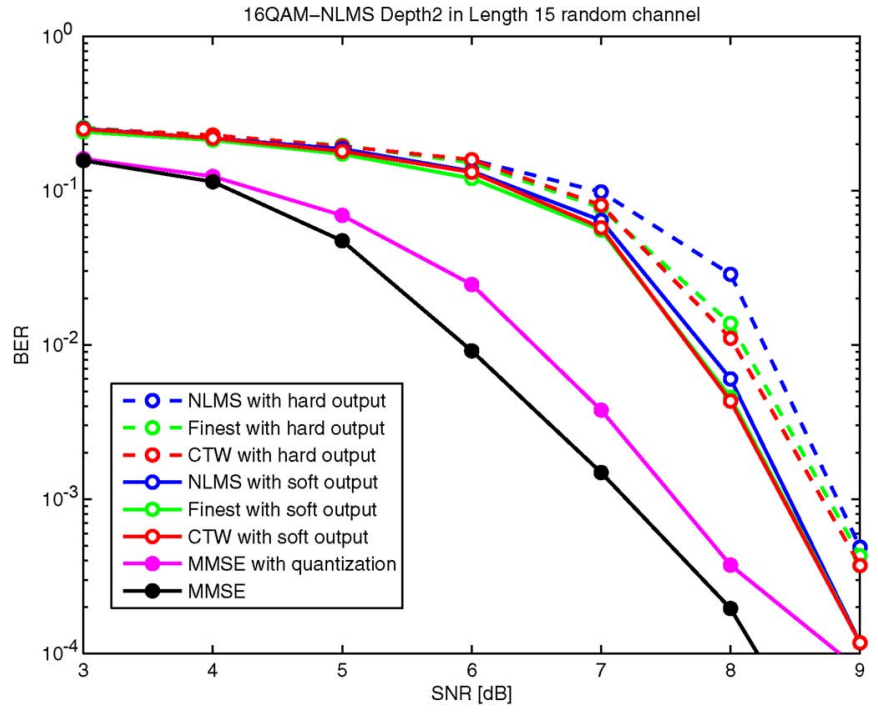

Fig. 13. BER comparison in the case of $16 \mathrm{QAM}$ at the 7 th turbo iteration in a randomly generated channel with length 15 .

data to train all the node filters, the context tree algorithm favors models with better performance. Note that at each iteration, we reset node probabilities $A_{\rho}(t)=1$ since a new tree is constructed using clustering.

As the next set of experiments, we perform BER performance comparison under 16QAM modulation with the time invariant ISI channel, $\boldsymbol{h}_{2}$. The BER results are plotted in Fig. 12, where the NLMS TREQ is used with $\mu=0.05$. We observe that BERs of adaptive algorithms with soft decision data or data-aided adaptive algorithms are close to the ideal MMSE equalizer. In the data-aided mode, the CTW algorithm is better than the other adaptive algorithms. To show the performance in a long delay spread channel, we performed experiments on a randomly generated channel of length 15 and provide the BER performance in Fig. 13. We observe similar performance improvement with the CTW algorithm in BER for this randomly generated channel as expected from our derivations. 


\section{CONCLUSION}

In this paper, we introduced an adaptive nonlinear turbo equalization algorithm using context trees to model the nonlinear dependency of the linear MMSE equalizer on the soft information generated from the decoder. We use the CTW algorithm to partition the space of variances, which are time dependent and generated from the soft information. We demonstrate that the algorithm introduced asymptotically achieves the performance of the best piecewise linear model defined on this context tree with a computational complexity only of the order of an ordinary linear equalizer. We also demonstrate the convergence of the MSE of the CTW algorithm to the MSE of the linear minimum MSE estimator as the depth of the context tree and the data length increase.

\section{APPENDIX A}

To calculate the difference between the MSE of the equalizer in (9) and the MSE of the linear MMSE equalizer in (2), we start with

$$
\begin{aligned}
\| \boldsymbol{w}_{k, o}^{T} & \boldsymbol{H}_{r} \boldsymbol{Q}(t) \boldsymbol{H}_{r}^{H} \boldsymbol{w}_{k, o}^{*}+\sigma_{n}^{2} \boldsymbol{w}_{k, o}^{T} \boldsymbol{w}_{k, o}^{*} \\
& -\left[1-\boldsymbol{v}^{H}\left(\sigma_{n}^{2} \boldsymbol{I}+\boldsymbol{H}_{r} \boldsymbol{Q}(t) \boldsymbol{H}_{r}^{H}+\boldsymbol{v} \boldsymbol{v}^{H}\right)^{-1} \boldsymbol{v}\right] \| \\
= & \boldsymbol{w}_{k, o}^{T} \boldsymbol{H}_{r} \Delta \tilde{\boldsymbol{Q}}_{k} \boldsymbol{H}_{r}^{H} \boldsymbol{w}_{k, o}^{*} \\
+ & {\left[1-\boldsymbol{v}^{H}\left(\sigma_{n}^{2} \boldsymbol{I}+\boldsymbol{H}_{r} \tilde{\boldsymbol{Q}}_{k} \boldsymbol{H}_{r}^{H}+\boldsymbol{v} \boldsymbol{v}^{H}\right)^{-1} \boldsymbol{v}\right] } \\
& -\left[1-\boldsymbol{v}^{H}\left(\sigma_{n}^{2} \boldsymbol{I}+\boldsymbol{H}_{r} \boldsymbol{Q}(t) \boldsymbol{H}_{r}^{H}+\boldsymbol{v} \boldsymbol{v}^{H}\right)^{-1} \boldsymbol{v}\right] \\
= & \boldsymbol{w}_{k, o}^{T} \boldsymbol{H}_{r} \Delta \tilde{\boldsymbol{Q}}_{k} \boldsymbol{H}_{r}^{H} \boldsymbol{w}_{k, o}^{*} \\
& +\boldsymbol{v}^{H}\left[\left(\boldsymbol{M}+\boldsymbol{H}_{r} \Delta \tilde{\boldsymbol{Q}}_{k} \boldsymbol{H}_{r}^{H}\right)^{-1}-\boldsymbol{M}^{-1}\right] \boldsymbol{v},
\end{aligned}
$$

where $\Delta \tilde{\boldsymbol{Q}}_{k} \triangleq \boldsymbol{Q}(t)-\tilde{\boldsymbol{Q}}_{k}$ (and the time index in $\Delta \tilde{\boldsymbol{Q}}_{k}$ is omitted for presentation purposes) and $\boldsymbol{M} \triangleq\left(\sigma_{n}^{2} \boldsymbol{I}+\boldsymbol{H}_{r} \tilde{\boldsymbol{Q}}_{k} \boldsymbol{H}_{r}^{H}+\boldsymbol{v} \boldsymbol{v}^{H}\right)$. To simplify the second term in (21), we use the first order expansion from the Lemma in the last part of Appendix A to yield

$$
\begin{aligned}
\boldsymbol{v}^{H} & \left(\boldsymbol{M}+\boldsymbol{H}_{r} \Delta \tilde{\boldsymbol{Q}}_{k} \boldsymbol{H}_{r}^{H}\right)^{-1} \boldsymbol{v} \\
= & \boldsymbol{v}^{H} \boldsymbol{M}^{-1} \boldsymbol{v}+\operatorname{tr}\left\{\nabla_{\Delta \tilde{\boldsymbol{Q}}_{k}}^{H}\left[\boldsymbol{v}^{H}\left(\boldsymbol{M}+\boldsymbol{H}_{r} \Delta \tilde{\boldsymbol{Q}}_{k} \boldsymbol{H}_{r}^{H}\right)^{-1} \boldsymbol{v}\right]\right. \\
& \left.\left.\quad\right|_{\Delta \tilde{\boldsymbol{Q}}_{k}=\mathbf{0}} \Delta \tilde{\boldsymbol{Q}}_{k}\right\} \\
& +O\left(\operatorname{tr}\left[\Delta \tilde{\boldsymbol{Q}}_{k}^{H} \Delta \tilde{\boldsymbol{Q}}_{k}\right]\right) \\
= & \boldsymbol{v}^{H} \boldsymbol{M}^{-1} \boldsymbol{v}+\operatorname{tr}\left(\boldsymbol{M}^{-1} \boldsymbol{v} \boldsymbol{v}^{H} \boldsymbol{M}^{-1} \Delta \tilde{\boldsymbol{Q}}_{k}\right) \\
& +O\left(\operatorname{tr}\left[\Delta \tilde{\boldsymbol{Q}}_{k}^{H} \Delta \tilde{\boldsymbol{Q}}_{k}\right]\right)
\end{aligned}
$$

around $\Delta \tilde{\boldsymbol{Q}}_{k}=\mathbf{0}$. Hence using (23) in (21) yields

$$
\begin{aligned}
\boldsymbol{w}_{k, o}^{T} & \boldsymbol{H}_{r} \Delta \tilde{\boldsymbol{Q}}_{k} \boldsymbol{H}_{r}^{H} \boldsymbol{w}_{k, o}^{*} \\
& +\boldsymbol{v}^{H}\left[\left(\boldsymbol{M}+\boldsymbol{H}_{r} \Delta \tilde{\boldsymbol{Q}}_{k} \boldsymbol{H}_{r}^{H}\right)^{-1}-\boldsymbol{M}^{-1}\right] \boldsymbol{v} \\
= & \boldsymbol{w}_{k, o}^{T} \boldsymbol{H}_{r} \Delta \tilde{\boldsymbol{Q}}_{k} \boldsymbol{H}_{r}^{H} \boldsymbol{w}_{k, o}^{*}+\operatorname{tr}\left(\boldsymbol{M}^{-1} \boldsymbol{v} \boldsymbol{v}^{H} \boldsymbol{M}^{-1} \Delta \tilde{\boldsymbol{Q}}_{k}\right) \\
& +O\left(\left\|\boldsymbol{q}(t)-\tilde{\boldsymbol{q}}_{k}\right\|^{2}\right) \leq O\left(\left\|\boldsymbol{q}(t)-\tilde{\boldsymbol{q}}_{k}\right\|^{2}\right)
\end{aligned}
$$

where the last line follows from the Schwartz inequality.
Lemma: We have [21]

$$
\begin{aligned}
& \nabla_{\Delta \tilde{\boldsymbol{Q}}_{k}} \boldsymbol{v}^{H}\left(\boldsymbol{M}+\boldsymbol{H}_{r} \Delta \tilde{\boldsymbol{Q}}_{k} \boldsymbol{H}_{r}^{H}\right)^{-1} \boldsymbol{v} \\
& =\left(\boldsymbol{M}+\boldsymbol{H}_{r} \Delta \tilde{\boldsymbol{Q}}_{k} \boldsymbol{H}_{r}^{H}\right)^{-1} \boldsymbol{v} \boldsymbol{v}^{H}\left(\boldsymbol{M}+\boldsymbol{H}_{r} \Delta \tilde{\boldsymbol{Q}}_{k} \boldsymbol{H}_{r}^{H}\right)^{-1} .
\end{aligned}
$$

Proof: To get the gradient of $\left(\boldsymbol{M}+\boldsymbol{H}_{r} \Delta \tilde{\boldsymbol{Q}}_{k} \boldsymbol{H}_{r}^{H}\right)^{-1}$ with respect to $\Delta \tilde{\boldsymbol{Q}}_{k}$, we differentiate the identity $\left(\boldsymbol{M}+\boldsymbol{H}_{r} \Delta \tilde{\boldsymbol{Q}}_{k} \boldsymbol{H}_{r}^{H}\right)^{-1}\left(\boldsymbol{M}+\boldsymbol{H}_{r} \Delta \tilde{\boldsymbol{Q}}_{k} \boldsymbol{H}_{r}^{H}\right)=\boldsymbol{I}$ with respect to $\left(\Delta \tilde{\boldsymbol{Q}}_{k}\right)_{a, b}$, i.e., the $a$ th and $b$ th element of the matrix $\Delta \tilde{\boldsymbol{Q}}_{k}$ and obtain

$$
\begin{aligned}
\partial\left(\boldsymbol{M}+\boldsymbol{H}_{r} \Delta \tilde{\boldsymbol{Q}}_{k} \boldsymbol{H}_{r}^{H}\right)^{-1} \partial\left(\Delta \tilde{\boldsymbol{Q}}_{k}\right)_{a, b}\left(\boldsymbol{M}+\boldsymbol{H}_{r} \Delta \tilde{\boldsymbol{Q}}_{k} \boldsymbol{H}_{r}^{H}\right) \\
+\left(\boldsymbol{M}+\boldsymbol{H}_{r} \Delta \tilde{\boldsymbol{Q}}_{k} \boldsymbol{H}_{r}^{H}\right)^{-1}\left(\boldsymbol{H}_{r} \boldsymbol{e}_{a} \boldsymbol{e}_{b}^{T} \boldsymbol{H}_{r}^{H}\right)=\mathbf{0}
\end{aligned}
$$

where $\boldsymbol{e}_{a}$ is a vector of all zeros except a single 1 at $a$ th entry. This yields

$$
\begin{aligned}
& \frac{\partial \boldsymbol{v}^{H}\left(\boldsymbol{M}+\boldsymbol{H}_{r} \Delta \tilde{\boldsymbol{Q}}_{k} \boldsymbol{H}_{r}^{H}\right)^{-1} \boldsymbol{v}}{\partial\left(\Delta \tilde{\boldsymbol{Q}}_{k}\right)_{a, b}} \\
& =\boldsymbol{v}^{H}\left(\boldsymbol{M}+\boldsymbol{H}_{r} \Delta \tilde{\boldsymbol{Q}}_{k} \boldsymbol{H}_{r}^{H}\right)^{-1} \\
& \times \boldsymbol{H}_{r} \boldsymbol{e}_{a} \boldsymbol{e}_{b}^{T} \boldsymbol{H}_{r}^{H}\left(\boldsymbol{M}+\boldsymbol{H}_{r} \Delta \tilde{\boldsymbol{Q}}_{k} \boldsymbol{H}_{r}^{H}\right)^{-1} \boldsymbol{v} \\
& =\operatorname{tr}\left\{\boldsymbol{e}_{b} \boldsymbol{H}_{r}^{H}\left(\boldsymbol{M}+\boldsymbol{H}_{r} \Delta \tilde{\boldsymbol{Q}}_{k} \boldsymbol{H}_{r}^{H}\right)^{-1}\right. \\
& \left.\times \boldsymbol{v} \boldsymbol{v}^{H}\left(\boldsymbol{M}+\boldsymbol{H}_{r} \Delta \tilde{\boldsymbol{Q}}_{k} \boldsymbol{H}_{r}^{H}\right)^{-1} \boldsymbol{H}_{r} \boldsymbol{e}_{a}\right\},
\end{aligned}
$$

which yields the result in (25) since (26) is the $(b, a)$ th element of the matrix in (25).

\section{APPENDIX B}

Outline of the proof of the theorem 2: The proof of the theorem follows the proof of the Theorem 2 of [19] and Theorem 1 of [29]. Hence, we mainly focus on differences.

Suppose we hypothetically construct all piecewise linear equalizers $\hat{x}_{\Gamma_{i}}(t), i=1, \ldots, m$ defined on the context tree and compute certain weights for all $t$

$$
c_{i}(t) \triangleq \frac{2^{-C\left(\Gamma_{i}\right)} \exp \left\{-a \sum_{r=1}^{t-1}\left[x(r)-\hat{x}_{\Gamma_{i}}(r)\right]^{2}\right\}}{\sum_{j=1}^{m} 2^{-C\left(\Gamma_{j}\right)} \exp \left\{-a \sum_{r=1}^{t-1}\left[x(r)-\hat{x}_{\Gamma_{j}}(r)\right]^{2}\right\}},
$$

where $0<C\left(\Gamma_{j}\right) \leq 2 K_{j}-1$ are constants that are used only for proof purposes such that $\sum_{k=1}^{m} 2^{-C\left(\Gamma_{j}\right)}=1[15]$ and $a$ is a positive constant set to $a=\frac{1}{2}=\frac{1}{2|\max \{x(t)\}|^{2}}$ [29]. Note that the weights defined in (27) are normalized functions of the performance of each $\hat{x}_{\Gamma_{i}}(t)$ on the observed data so far, i.e., the better performing piecewise linear equalizers would have higher weights. At each time $t$, if we define a weighted equalizer

$$
\hat{x}(t) \triangleq \sum_{k=1}^{m} c_{k}(t) \hat{x}_{\Gamma_{k}}(t),
$$

then it follows from Theorem 1 of [29] that the performance of the weighted equalizer $\hat{x}(t)$ satisfies

$$
\sum_{t=1}^{n}[x(t)-\hat{x}(t)]^{2} \leq \sum_{t=1}^{n}\left[x(t)-\hat{x}_{\Gamma_{i}}(t)\right]^{2}+2 K_{i}-1,
$$


for all $i=1, \ldots, m$. In this sense, $\hat{x}(t)$ is the desired $\hat{x}_{\mathrm{ctw}}(t)$, i.e., it achieves the performance of the best piecewise equalizer among $m$ piecewise linear equalizers defined on the context tree. The proof that (28) satisfies (29) is based on defining universal probabilities, using telescoping and certain convexity arguments, however, straightforward and is not repeated here. However, even if we have the result in (29), note that $\hat{x}(t)$ requires the outputs of doubly exponential number $m$ algorithms and computes $m$ performance based weights in (27), which is computationally infeasible for large $m$. We next show that such a doubly exponential number of algorithms and weights can be efficiently calculated on the context tree, i.e., the weighted summation in (29) can be efficiently calculated because of the tree structure.

To circumvent this problem, we first observe that any $\hat{x}_{\Gamma_{i}}(t)$ defined in the binary tree is constructed from a subset of $2^{D+1}$ 1 node equalizers $\hat{x}_{\rho}(t), \rho \in\left\{1, \ldots, 2^{D+1}-1\right\}$. At each time $t$, for each partition $\Gamma_{i}$, we find the corresponding node that $\boldsymbol{q}(t)$ belongs to and repeat the output of the $\hat{x}_{\rho}(t)$ corresponding to that node as the output of $\hat{x}_{\Gamma_{i}}(t)$. However, at each time $t$, due to the tree structure of the partitions, $\boldsymbol{q}(t)$ can only belong to $D+1$ regions or nodes on the tree. As an example, if $\boldsymbol{q}(t)$ belongs to the left-left hand child, then it also belongs to the left-hand child and the root node. For such a $\boldsymbol{q}(t)$, all $m$ piecewise linear equalizers would equal to one of these three node outputs. In this sense, although we have $m$ piecewise equalizers in (27), these equalizers can only output $D+1$ distinct values, i.e., the outputs of the nodes that $\boldsymbol{q}(t)$ belongs. Hence, at each time $t$, $\hat{x}(t)$ is constructed as a weighted sum of only $D+1$ distinct node predictions. Then, all the weights in (27) with the same node predictions can be merged.

To be able to define such a merging with computational complexity only linear in $D$, as shown in [19], we define certain functions of performance for each node as $A_{\rho}(t), B_{\rho}(t)$ that are initialized in (line A) and updated in (line C), (line D), (line E) of Fig. 7. These variables measure the performance as in (27), where

$$
B_{\rho}(t) \triangleq \exp \left(-\frac{1}{2 a} \sum_{t=1}^{n_{\rho}}\left(x(t)-\hat{x}_{\rho}(t)\right)^{2}\right)
$$

is the performance of each node piecewise linear equalizer on the data observed so far, $n_{\rho}$ is the nodes assigned to the $\rho$ and

$$
A_{\rho}(t) \triangleq A_{\rho_{l}}(t) A_{\rho_{r}}(t)+\frac{1}{2} B_{\rho}(t)
$$

is the accumulated weight recursively calculated for each $\hat{x}_{\rho}(t)$ at the inner nodes, where $A_{\rho_{l}}(t)$ and $A_{\rho_{r}}(t)$ are the weights for each child node. Then, the corresponding $\hat{x}(t)$ can be defined as a merged summation of $D+1$ node outputs as

$$
\hat{x}(t)=\sum_{l=1}^{D+1} \beta_{k}(t) \hat{x}_{\boldsymbol{l}(l)}(t),
$$

where $\boldsymbol{l}$ contains the nodes that $\boldsymbol{q}(t)$ belongs to and $\beta_{k}(t)$ are calculated as shown in (line B) of Fig. 7 based on $A_{\rho}(t)$ and $B_{\rho}(t)$. Hence, the desired equalizer is given by

$$
\hat{x}_{\mathrm{ctw}}(t)=\sum_{l=1}^{D+1} \beta_{k}(t) \hat{x}_{\boldsymbol{l}(l)}(t),
$$

which requires computing $D+1$ node estimations and updates only $D+1$ node equalizers at each time $t$ and store $2^{D+1}-1$ node weights. This completes the outline of the proof of (13).

To get the corresponding result in (14), we define the node predictors as the LS predictors such that

$$
\begin{aligned}
{\left[\boldsymbol{w}_{\rho}(t) \boldsymbol{f}_{\rho}(t)\right]^{T} } & =\boldsymbol{M}^{-1}(t-1) \boldsymbol{p}(t-1), \boldsymbol{M}(t-1) \\
& \triangleq\left(\sum_{r=1}^{t} \boldsymbol{d}(r-1) \boldsymbol{d}(r-1)^{T} s_{\rho}(r)+\delta \boldsymbol{I}\right)
\end{aligned}
$$

and $\boldsymbol{p}(t-1) \triangleq \sum_{r=1}^{t-1} Q(\hat{y}(r)) \boldsymbol{d}(r-1) s_{\rho}(r)$, where $\boldsymbol{d}(r) \triangleq$ $[\boldsymbol{y}(r) \overline{\boldsymbol{x}}(r)]^{T}, s_{\rho}(r)$ is the indicator variable for node $\rho$, i.e., $s_{\rho}(r)=1$ if $\boldsymbol{q}(r) \in V_{\rho}$ otherwise $s_{\rho}(r)=0$. The affine predictor in (30) is a least squares predictor that trains only on the observed data $\{y(t)\}$ and $\{\bar{x}(t)\}$ that belongs to that node, i.e., that falls into the region $V_{\rho}$. Note that the update in (30) can be implemented with $O(1)$ computations using fast inversion methods [30]. For each node $\rho$, the RLS algorithm is shown to achieve the excess loss

$$
\begin{aligned}
& \sum_{t=1}^{n_{\rho}}\left\{\left[x(t)-\hat{x}_{\rho}(t)\right]^{2}\right\} \\
& \leq \min _{\boldsymbol{w}_{\rho} \in \mathbb{C}^{N}, \boldsymbol{f}_{\rho} \in \mathbb{C}^{N+M-1}} \sum_{t=1}^{n_{\rho}}\left\{\left[x(t)-\boldsymbol{w}_{\rho}^{T} \boldsymbol{y}(t)-\boldsymbol{f}_{\rho}^{T} \overline{\boldsymbol{x}}(t)\right]^{2}\right\} \\
& \quad+O\left((N+M) \ln \left(n_{\rho}\right)\right)
\end{aligned}
$$

where $n_{\rho}$ is the number of samples that fall into the node $\rho$. Hence, application of this results to each node predictor in (29) yields the result in (14) as shown in [31].

\section{REFERENCES}

[1] C. Douillard, M. Jezequel, C. Berrou, A. Picart, P. Didier, and A Glavieux, "Iterative correction of inter-symbol interference: Turbo equalization," Eur. Trans. Telecommun., vol. 6, no. 5, pp. 507-511, Sep.-Oct. 1995.

[2] J. Hagenauer, "The turbo principle: Tutorial introduction and state of the art," in Proc. Int. Symp. Turbo Codes, Brest, France, 1997, pp. 1-11.

[3] C. Berrou and A. Glavieux, "Near optimum error correcting coding and decoding: Turbo codes," IEEE Trans. Commun., vol. 44, pp. 1261-1271, Oct. 1996.

[4] A. Glavieux, C. Laot, and J. Labat, "Turbo equalization over a frequency selective channel," in Proc. Int. Symp. Turbo Codes, Brest, France, 1997, pp. 96-102.

[5] M. Tüchler, R. Koetter, and A. C. Singer, "Turbo equalization: Principles and new results," IEEE Trans. Commun., vol. 50, no. 5, pp. 754-767, May 2002.

[6] S. Song, A. C. Singer, and K.-M. Sung, "Soft input channel estimation for turbo equalization," IEEE Trans. Signal Process., vol. 52, no. 10, pp. 2885-2894, Oct. 2004.

[7] C. Studer, S. Fateh, and D. Seethaler, "Asic implementation of softinput soft-output MIMO detection using parallel interference cancellation," IEEE J. Solid-State Circuits, vol. 56, no. 7, pp. 1754-1765, Jul. 2011.

[8] J. Proakis, Digital Communications. New York, NY, USA: McGrawHill, 1995.

[9] C. Laot, R. Le Bidan, and D. Leroux, "Low-complexity MMSE turbo equalization: A possible solution for EDGE," IEEE Trans. Wireless Commun., vol. 4, no. 3, pp. 965-974, May 2005.

[10] J. W. Choi, R. J. Drost, A. C. Singer, and J. Preisig, "Iterative multichannel equalization and decoding for high frequency underwater acoustic communications," in Proc. IEEE Sensor Array Multichannel Signal Process. Workshop, 2008, pp. 127-130.

[11] A. C. Singer, J. K. Nelson, and S. S. Kozat, "Signal processing for underwater acoustic communications," IEEE Commun. Mag., vol. 47, no. 1, pp. 90-96, Jan. 2009. 
[12] R. Le Bidan, "Turbo-equalization for bandwith-efficient digital communications over frequency-selective channels," Ph.D. dissertation, Institut TELECOM/TELECOM, Bretagne, France, 2003.

[13] M. Tüchler, "Iterative Equalization Using Priors," M.Sc. thesis, Univ. of Illinois at Urbana-Champaign, Urbana, IL, USA, 2000.

[14] M. Tüchler, A. C. Singer, and R. Koetter, "Minimum mean squared error equalization using priors," IEEE Trans. Signal Process., vol. 50, no. 3, pp. 673-683, Mar. 2002.

[15] F. M. J. Willems, "Coding for a binary independent piecewise-identically-distributed source," IEEE Trans. Inf. Theory, vol. 42, pp. 2210-2217, Nov. 1996.

[16] S. S. Kozat and A. C. Singer, "Universal switching linear least squares prediction,” IEEE Trans. Signal Process., vol. 56, pp. 189-204, Jan. 2008.

[17] D. P. Helmbold and R. E. Schapire, "Predicting nearly as well as the best pruning of a decision tree," Mach. Learn., vol. 27, no. 1, pp. 51-68, Apr. 1997.

[18] E. Takimoto, A. Maruoka, and V. Vovk, "Predicting nearly as well as the best pruning of a decision tree through dyanamic programming scheme," Theoretic. Comput. Sci., vol. 261, pp. 179-209, June 2001.

[19] S. S. Kozat, A. C. Singer, and G. C. Zeitler, "Universal piecewise linear prediction via context trees," IEEE Trans. Signal Process., vol. 55, pp. 3730-3745, Jul. 2007.

[20] Y. Yilmaz and S. S. Kozat, "Competitive randomized nonlinear prediction under additive noise," IEEE Signal Process. Lett., vol. 17, no. 4, pp. 335-339, Apr. 2010.

[21] A. Graham, Kronecker Products and Matrix Calculus: With Applications. Chichester, U.K.: Ellis Horwood, 1981.

[22] S. Lee, A. C. Singer, and N. R. Shanbhag, "Linear turbo equalization analysis via BER transfer and EXIT charts," IEEE Trans. Signal Process., vol. 53, pp. 2883-2897, Aug. 2005

[23] O. J. J. Michel, A. O. Hero, and A. E. Badel, "Tree structured nonlinear signal modeling and prediction," IEEE Trans. Signal Process., pp. 3027-3041, Nov. 1999.

[24] A. Gersho and R. M. Gray, Vector Quantization and Signal Compression, ser. Springer Int. Series in Engineering and Computer Science. New York, NY, USA: Springer, 1992

[25] S. Haykin, Adaptive Filter Theory. Englewood Cliffs, NJ, USA: Prentice-Hall, 1996.

[26] K. Kim, J. W. Choi, A. C. Singer, and K. Kim, "A new adaptive turbo equalizer with soft information classification," in Proc. Int. Conf. Acoust., Speech, Signal Process., Dallas, TX, USA, 2010, pp. 3206-3209.

[27] D. P. Chaudhuri, C. A. Murthy, and B. B. Chaudhuri, "A modified metric to compute distance," Pattern Recognit., vol. 25, no. 7, pp. 667-677, Jul. 1992.

[28] A. H. Sayed, Fundamentals of Adaptive Filtering. New York, NY, USA: Wiley, 2003.

[29] A. C. Singer and M. Feder, "Universal linear prediction by model order weighting," IEEE Trans. Signal Process., vol. 47, no. 10, pp. 2685-2699, Oct. 1999.

[30] J. Cioffi and T. Kailath, "Fast recursive least squares traversal filters for adaptive filtering," IEEE Trans. Acoust., Speech, Signal Process., vol. 32, pp. 304-337, Nov. 1984.

[31] N. Merhav and M. Feder, "Universal schemes for sequential decision from individual sequences," IEEE Trans. Inf. Theory, vol. 39, no. 4, pp. 1280-1292, Jul. 1993.

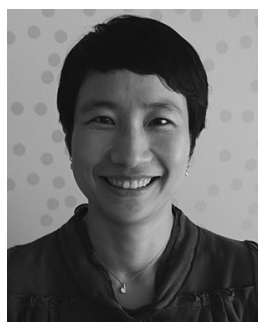

Kyeongyeon Kim (M'07) received the B.S., M.S., and Ph.D. degrees in electrical engineering from Yonsei University, Seoul, Korea, in 2001, 2003 and 2007, respectively.

After graduation, she was a Postdoctoral Fellow in Purdue University, West Lafayette, IN, USA, from 2007 to 2008 and in the University of Illinois at Urbana-Champaign, USA, from 2008 to 2010. Since 2010, she has been with Samsung Advanced Institute of Technology (SAIT), Samsung Electronics, Yongin-si, Korea, and participated in low-power receiver algorithm design and software implementation of wireless communication and broadcasting systems. Her research interests include signal processing for wireless/underwater communication and broadcasting systems, array signal processing, and communication system anaysis and design.

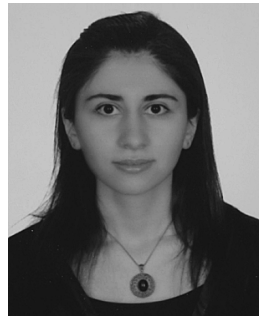

Nargiz Kalantarova was born on October 29, 1985, in Baku, Azerbaijan. She received the B.S. degrees in both electrical engineering and mathematics from Bogazici University, Istanbul, Turkey, in 2008.

Currently, she is a Research Assistant and is working toward the M.S. degree at the Electrical and Computer Engineering in Koc University, Istanbul, Turkey. Her research interests include statistical signal processing, digital communications, and optimization.

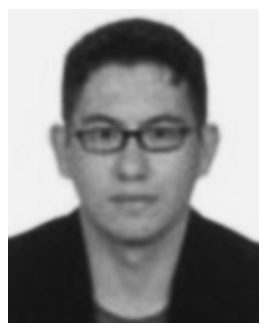

Suleyman Serdar Kozat (SM'11) received the B.S. degree with full scholarship and high honors from Bilkent University, Ankara, Turkey, and the M.S. and $\mathrm{Ph} . \mathrm{D}$. degrees in electrical and computer engineering from the University of Illinois at Urbana Champaign, Urbana, IL, USA, in 2001 and 2004, respectively.

After graduation, he joined IBM Research, T. J. Watson Research Lab, Yorktown, NY, USA, as a Research Staff Member in the Pervasive Speech Technologies Group, where he focused on problems related to statistical signal processing and machine learning. While working on his Ph.D., he was also working as a Research Associate at Microsoft Research, Redmond, WA, USA, in the Cryptography and Anti-Piracy Group. He holds several patent inventions due to his research accomplishments at IBM Research and Microsoft Research. After serving as an Assistant Professor at Koc University, Istanbul, Turkey, he is currently an Assistant Professor (with the Associate Professor degree from Yuksek Ogretim Kurumu) at the Electrical And Electronics Department of Bilkent University. Overall, his research interests include signal processing, adaptive filtering, sequential learning, and machine learning.

Dr. Kozat has been serving as an Associate Editor for the IEEE TRANSACTIONS ON Signal PROCESSING (and he is currently the only Associate Editor in Turkey for this top ranking signal processing journal). $\mathrm{He}$ is the General Co-Chair for the IEEE Machine for Signal Processing, Istanbul, Turkey, 2013. He has been awarded the IBM Faculty Award by IBM Research in 2011, the Outstanding Faculty Award by Koc University in 2011 (granted the first time in 16 years), the Outstanding Young Researcher Award by the Turkish National Academy of Sciences in 2010, the ODTU Prof. Dr. Mustafa N. Parlar Research Encouragement Award in 2011, and holds Career Award by the Scientific Research Council of Turkey, 2009. He has also served in many Technical Committees of different international and national conferences and workshops. During his high school years, he won several scholarships and medals in international and national science and math competitions.

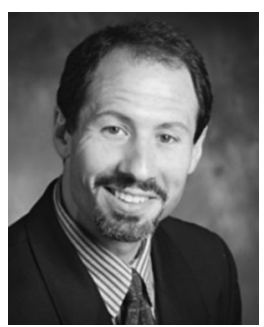

Andrew C. Singer (F'10) received the S.B., S.M., and $\mathrm{Ph} . \mathrm{D}$. degrees, all in electrical engineering and computer science, from the Massachusetts Institute of Technology (MIT), Cambridge, MA, USA.

Since 1998, he has been on the faculty of the Department of Electrical and Computer Engineering at the University of Illinois at Urbana-Champaign, IL, USA, where he is currently a Professor in the Electrical and Computer Engineering Department and the Coordinated Science Laboratory. During academic year 1996, he was a Postdoctoral Research Affiliate in the Research Laboratory of Electronics at MIT. From 1996 to 1998, he was a Research Scientist at Sanders, A Lockheed Martin Company, Manchester, NH, USA, where he designed algorithms, architectures, and systems for a variety of DOD applications. His research interests include signal processing and communication systems.

Dr. Singer was a Hughes Aircraft Masters Fellow, and was the recipient of the Harold L. Hazen Memorial Award for excellence in teaching in 1991. In 2000, he received the National Science Foundation CAREER Award; in 2001, he received the Xerox Faculty Research Award; and in 2002, he was named a Willett Faculty Scholar. He has served as an Associate Editor for the IEEE Transactions on Signal Processing and is a member of the MIT Educational Council and of Eta Kappa Nu and Tau Beta Pi. 